\title{
Circumventing the radiation pressure barrier in the formation of massive stars via disk accretion
}

\author{
Rolf Kuiper ${ }^{1,2}$ \\ kuiper@astro.uni-bonn.de \\ and \\ Hubert Klahr ${ }^{2}$ \\ and \\ Henrik Beuther ${ }^{2}$ \\ and \\ Thomas Henning ${ }^{2}$
}

\begin{abstract}
We present radiation hydrodynamics simulations of the collapse of massive pre-stellar cores. We treat frequency dependent radiative feedback from stellar evolution and accretion luminosity at a numerical resolution down to $1.27 \mathrm{AU}$. In the $2 \mathrm{D}$ approximation of axially symmetric simulations, it is possible for the first time to simulate the whole accretion phase (up to the end of the accretion disk epoch) for the forming massive star and to perform a broad scan of the parameter space. Our simulation series show evidently the necessity to incorporate the dust sublimation front to preserve the high shielding property of massive accretion disks. While confirming the upper mass limit of spherically symmetric accretion, our disk accretion models show a persistent high anisotropy of the corresponding thermal radiation field. This yields to the growth of the highest-mass stars ever formed in multi-dimensional radiation hydrodynamics simulations, far beyond the upper mass limit of spherical accretion. Non-axially symmetric effects are not necessary to sustain accretion. The radiation pressure launches a stable bipolar outflow, which grows in angle with time as presumed from observations. For an initial mass of the pre-stellar host core of $60,120,240$, and $480 \mathrm{M}_{\odot}$ the masses of the final stars formed in our simulations add up to $28.2,56.5,92.6$, and at least $137.2 \mathrm{M}_{\odot}$ respectively.
\end{abstract}

Subject headings: Accretion, accretion disks - Hydrodynamics — Methods: numerical - Radiative transfer - Stars: formation - Stars: massive

\footnotetext{
${ }^{1}$ Argelander Institute for Astronomy, Bonn University, Auf dem Hügel 71, D-53121 Bonn, Germany

${ }^{2}$ Max Planck Institute for Astronomy, Königstuhl 17, D-69117 Heidelberg, Germany
} 


\section{Introduction}

The understanding of massive stars still suffers from the lack of a generally accepted formation scenario. Despite the strong limitations in observations of massive star forming regions compared to their low-mass counterpart, past studies obtained common features of star formation, suggesting that the formation of massive stars can in first order be treated analog to low-mass star formation. All epochs of the classical picture of star formation, such as gravitationally collapsing massive cores (e.g. Ho \& Haschick 1986; Keto et al. 1987; Zhang \& Ho 1997; Birkmann et al. 2007) and collimated jets as well as wide-angle bipolar outflows (e.g. Henning et al. 2000; Zhang et al. 2002, 2007; Wu et al. 2005; Beuther et al. 2005a) were observed. Even circumstellar disks, e.g. IRAS 20126+4104 (Cesaroni et al. 1997; Zhang et al. 1998; Cesaroni et al. 2005) and AFGL 490 (Harvey et al. 1979; Torrelles et al. 1986; Chini et al. 1991; Davis et al. 1998; Lyder et al. 1998; Schreyer et al. 2002, 2006), or rather large scale toroid (Beltrán et al. 2004, 2005) and flattened rotating structures (Beuther et al. 2005b; Beuther \& Walsh 2008) could be revealed. Reviews of observations related to a proposed picture of evolutionary sequences of massive star formation are given in Beuther et al. (2007) or Zinnecker \& Yorke (2007).

Previous theoretical models focused on different points of view, namely the competitive accretion (Bonnell et al. 1998; Bonnell \& Bate 2002; Bonnell et al. 2004; Bate 2009b,a) and the turbulent core model (McKee \& Tan 2003), but they agree on the formation of accretion disks.

If the formation of high-mass stars is therefore treated as a scaled-up version of low-mass star formation, a special feature of these high-mass proto-stars is the interaction of the accretion flow with the strong irradiation emitted by the newborn stars due to their short Kelvin-Helmholtz contraction timescale (Shu et al. 1987). Previous one-dimensional studies (e.g. Larson \& Starrfield 1971; Kahn 1974; Yorke \& Krügel 1977; Wolfire \& Cassinelli 1987; Edgar \& Clarke 2004) agree on the fact that the growing radiation pressure potentially stops and reverts the accretion flow onto a massive star yielding an upper mass limit of approximately $40 \mathrm{M}_{\odot}$.

But this radiative impact strongly depends on the geometry of the stellar environment (Nakano 1989). The possibility was suggested to overcome this radiation pressure barrier via the formation of a long-living massive circumstellar disk, which forces the generation of a strong anisotropic feature of the thermal radiation field. Earlier investigations by Yorke \& Sonnhalter (2002) tried to identify such an anisotropy, which they called the "flashlight effect", in two-dimensional axially and midplane symmetric radiation hydrodynamics simulations, similar to our own. Their simulations show an early end of the disk accretion phase shortly after its formation due to strong radiation pressure. The final star masses are only marginally higher than the mass limit in spherical symmetry, even if the frequency dependence of the radiation is considered. The radiative feedback was treated under the flux limited diffusion approximation (hereafter called FLD), but computed for several frequency bins. Due to the high computational cost of the frequency dependent FLD solver in Yorke \& Sonnhalter (2002), it was unfortunately not possible to study the reason for the early fate of the disk accretion phase in detail. Krumholz et al. (2009) stated that the circumstellar 
disk in the simulations of Yorke \& Sonnhalter (2002) lost its shielding property because the disk region cannot be fed in axially symmetric configuration. Contrary to the stable radiation pressure driven outflows in Yorke \& Sonnhalter (2002), they discovered in their own three-dimensional (frequency averaged) radiation hydrodynamics simulations (Krumholz et al. 2007, 2009) an instability in the outflow region, leading to further accretion onto the disk. They propose that this so-called "3D radiative Rayleigh-Taylor instability" requires non-axially symmetric modes to occur (Krumholz et al. 2009) and that radiation pressure therefore cannot halt a flow of gas and dust in any direction. This explanation suffers from a lack of physical arguments and requires further detailed investigation due to the fact that first the classical Rayleigh-Taylor instability is a twodimensional instability and secondly radiation cannot simply be treated as a fluid in general (as it is in the sense of a Rayleigh-Taylor instability). At least it remains unclear if this instability is the most important one. With the help of the unstable polar region, the most massive star in their simulations grows up to $41.5 \mathrm{M}_{\odot}$ with an ongoing accretion phase, as the simulation is not finished yet.

Contrary to this explanation of the short accretion phases in the two-dimensional simulations by Yorke \& Sonnhalter (2002), we demonstrate here in detail the need of including the radiation physics at the dust sublimation front of the forming star to compute the correct anisotropy of the re-emitted radiation by dust grains. Due to the huge sink cells used in the simulations by Yorke \& Sonnhalter (2002), the luminosity directly acts onto a disk region far beyond the actual dust sublimation front. The interaction of the radiation with the accretion flow at the dust sublimation front is therefore artificially shifted to the radius of the sink cell, where the circumstellar disk would originally be shielded from the direct stellar irradiation. In this region the optical depth of the IR flux in the radial direction is much smaller than at the realistic location of the dust sublimation front, yielding a much higher fraction of isotropy of the radiation field. As shown in the well-established spherical symmetric studies, this isotropic radiation field is able to stop and revert the accretion flow onto the forming star.

Our simulations focus on the accretion onto a single massive star in the center of the core. We incorporate the dust sublimation front of the forming star, resolve the vicinity of the star down to $1.27 \mathrm{AU}$, and evolve the system up to the order of $10^{5} \mathrm{yr}$ (ten times longer than ever studied before), including the whole disk accretion phase of the forming star. We scan the broad parameter space of numerical configurations as well as different initial conditions in several simulation series.

In the following Sect. 2, we describe the details of the self-gravity radiation hydrodynamics code in use. The physical initial conditions of the pre-stellar cores as well as the numerical configuration of the simulations are described in Sect. 3. In Sects. 4 and 5, we present the results of one- and twodimensional radiation hydrodynamics simulations of massive pre-stellar core collapses respectively, focusing on the radiative feedback onto the accretion flow while resolving the dust sublimation front. A discussion (Sect. 6) and summary of the most important results (Sect. 7) including a comparison of our results to the simulations by Yorke \& Sonnhalter (2002) and Krumholz et al. (2007, 2009), a discussion of our assumptions, as well as a brief outlook on the future direction of 
this research project, e.g. 3D simulations, close this publication.

\section{Physics and numerics}

In this section, we outline the ingredients and default numerical configuration of the self-gravity radiation hydrodynamics code we use to model the collapse of massive pre-stellar cores. The first Sect. 2.1 comprises the motivation for our choice of a grid in spherical coordinates and highlights the step forward in resolution we obtain in our simulations compared to previous research. The following two Sects. 2.2 and 2.3 describe the features and the configuration of the hydrodynamics solver including full tensor viscosity, for which we use the open source magneto-hydrodynamics code Pluto3 (Mignone et al. 2007). Further sections describe our newly developed modules for self-gravity (Sect. 2.4) and frequency dependent approximate radiation transport (Sect. 2.5). We close this section with the description of the pre-calculated, tabulated dust (Sect. 2.6) and stellar evolution model (Sect. 2.7) used in the simulations.

\subsection{Discretization of the computational domain}

In our simulations, we use a time independent grid in spherical coordinates with logarithmically increasing radial resolution towards the center. The usage of a radially increasing resolution towards the center guarantees the possibility to study the radiative feedback in the central core regions down to a minimum grid cell size of $\Delta r \times r \Delta \theta=1.27$ AU x 1.04 AU. An example of such a twodimensional grid is displayed in Fig. 1. This type of grid is well adapted for the analysis of the interaction of an accretion flow onto a massive star along with the stellar irradiation it generates, because the stellar gravity as well as the stellar radiative force are aligned with the radial coordinate axis. Furthermore, in contrast to e.g. cartesian coordinates, the usage of spherical coordinates guarantees a strict angular momentum conservation. The polar discretization $\Delta \theta$ of the grid is uniformly fixed and covers an angle of $\pi / 2$ from the top polar axis to the forming disk midplane, assuming midplane symmetry as in Yorke \& Sonnhalter (2002). The polar resolution $r \Delta \theta$ of the spherical grid automatically increases towards the regions of interest around the centrally forming star, where high resolution is desired. To even enhance this focus on the inner parts of the prestellar core and saving computational effort in the outer parts far away from the dust radiation interaction layer, we choose a logarithmically increasing radial resolution of the grid. Thus the non-adaptive grid setup impeded the study of potential fragmentation in the outer core regions. The radial resolution $\Delta r$ at a radius $r$ of a computational domain with $N_{r}$ grid cells in the radial direction is given by

$$
\Delta r(r)=r\left(10^{f}-1\right)
$$

with $f=\log \left(r_{\max } / r_{\min }\right) / N_{r}$, where $r_{\min }$ and $r_{\max }$ represent the inner and outer radius of the computational domain. A comparison of our achieved resolution to previous massive pre-stellar 

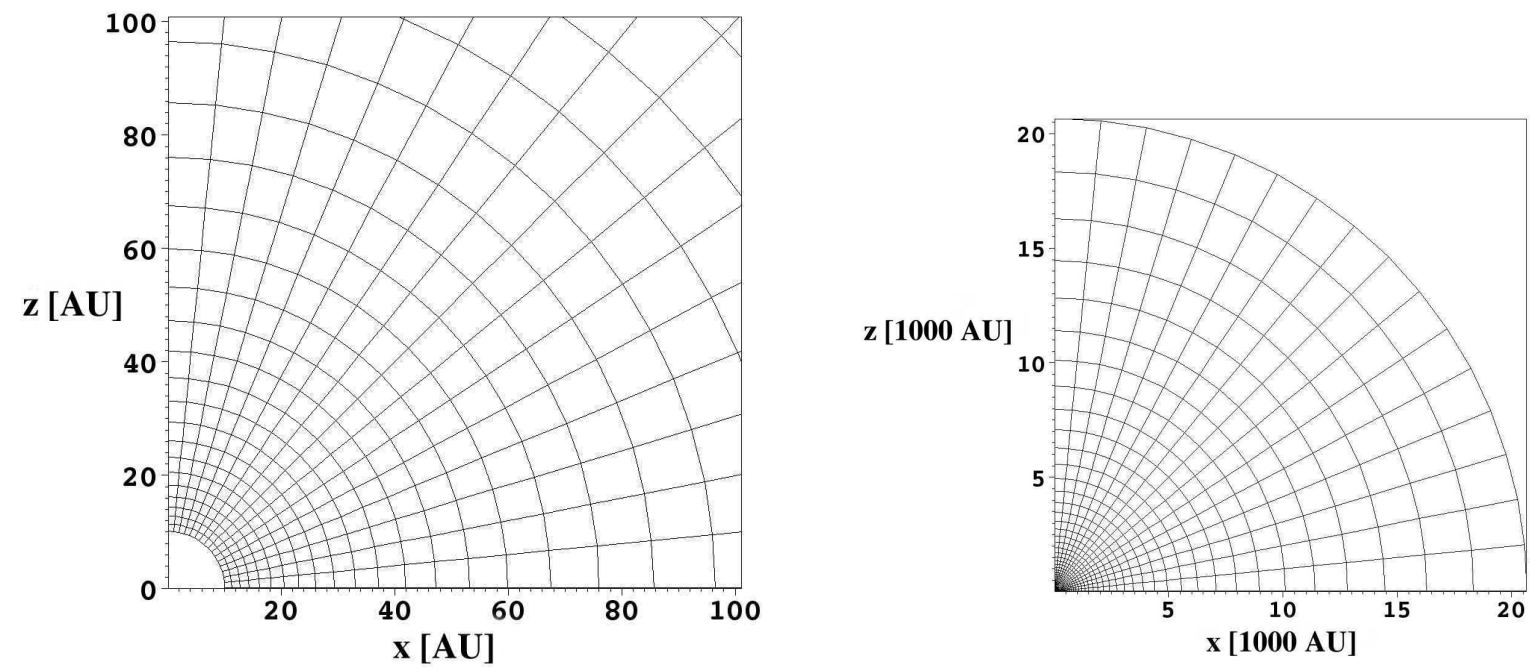

(a) Global image of the total computational domain up to (b) Zoom-in image of the central 100 AU x 100 AU. the outer radius of $r_{\max }=0.1 \mathrm{pc}$. The innermost cells have a resolution of $\Delta r \times \Delta \theta=1.27 \mathrm{AU} \times 1.04 \mathrm{AU}$.

Fig. 1.- : Two-dimensional grid (64 x 16) in spherical coordinates with logarithmically increasing radial resolution, a central sink cell of radius $r_{\min }=10 \mathrm{AU}$, and an outer boundary at $r_{\max }=0.1 \mathrm{pc}$. 
core collapse simulations by Yorke \& Sonnhalter (2002) and Krumholz et al. (2007, 2009) is given in Table 1.

The forming high-mass proto-stellar object at the center of the core is represented by a dedicated stellar evolution model (presented in Sect. 2.7) inside the central sink cell with radius $r_{\text {min }}$ at the origin of the coordinate system using pre-calculated stellar evolutionary tracks for accreting high-mass proto-stars (Hosokawa \& Omukai 2009).

\subsection{Hydrodynamics}

To follow the motion of the gas, we solve the equations of compressible hydrodynamics

$$
\begin{aligned}
\partial_{t} \rho+\vec{\nabla} \cdot(\rho \vec{u}) & =0 \\
\partial_{t}(\rho \vec{u})+\vec{\nabla}(\rho \vec{u} \vec{u}+P) & =\rho \vec{a} \\
\partial_{t} E+\vec{\nabla} \cdot((E+P) \vec{u}) & =\rho \vec{u} \cdot \vec{a}-\vec{\nabla} \vec{F}_{\text {tot }}
\end{aligned}
$$

with the acceleration source term $\vec{a}=\sum_{i} \vec{a}_{i}$, which includes the additionally considered physics to the equations of gas dynamics (Euler equations) such as shear viscosity $\left(\vec{a}_{1}\right)$, central gravity of the forming star $\left(\vec{a}_{2}\right)$, self-gravity $\left(\vec{a}_{3}\right)$, and radiation transport and stellar radiative feedback $\left(\vec{a}_{4}\right) . \vec{F}_{\text {tot }}$ denotes the flux of the total radiation energy density. These additional components are described in the following subsections. The evolution of the gas density $\rho$, velocity $\vec{u}$, pressure $P$, and total energy density $E$ is computed using the open source magneto-hydrodynamics code Pluto3 (Mignone et al. 2007).

Pluto is a high-order Godunov solver code, i.e. it uses a shock capturing Riemann solver within a conservative finite volume scheme. The numerical configuration of our simulations makes use of a Strang operator splitting scheme for the different dimensions (Strang 1968). Our default configuration consists further of a Harten-Lax-Van Leer (hll) Riemann solver and a so-called 'minmod' flux limiter using piecewise linear interpolation (plm) and a Runge-Kutta 2 (RK2) time integration, also known as the predictor-corrector-method, compare van Leer (1979). Therefore the total difference scheme is accurate to $2^{\text {nd }}$ order in time and space.

To close the system of Eqs. (2) to (4), we use an ideal gas equation of state

$$
P=(\gamma-1) E_{\text {int }},
$$

which relates the gas pressure $P$ to the internal energy $E_{\text {int }}=E-0.5 \rho u^{2}$. The adiabatic index $\gamma$ is set to $5 / 3$.

To limit the range of densities, the so-called floor value of the density is chosen to be $\rho_{0}=$ $10^{-21} \mathrm{~g} \mathrm{~cm}^{-3}$. This floor value occurs during the simulations only in regions where the radiation pressure driven outflow is depleting the density of the corresponding grid cells in the radially 
Table 1. Resolution of different radiation hydrodynamics simulations of a collapse of a slowly rotating massive pre-stellar core

\begin{tabular}{|c|c|c|c|}
\hline Authors & $\begin{array}{l}\text { Resolution in } \\
\text { lowest resolution }\end{array}$ & $\begin{array}{l}\mathrm{AU} \text { in regions of } \\
\text { highest resolution }\end{array}$ & $\begin{array}{c}\text { Radius of } \\
\text { sink cells (AU) }\end{array}$ \\
\hline Yorke \& Sonnhalter (2002) & $320^{2}$ & $80^{2}$ & 80 \\
\hline Krumholz et al. (2007) & $966^{3}$ & $7.5^{3}$ & $0-30$ \\
\hline Krumholz et al. (2009) & $645^{3}$ & $10^{3}$ & $0-40$ \\
\hline This study 1D & 1540 & 0.08 & 1.0 \\
\hline This study $2 \mathrm{D}$ & $2319 \times 1911$ & $1.27 \times 1.04$ & 10.0 \\
\hline
\end{tabular}

Note. - The simulations of Yorke \& Sonnhalter (2002) were performed on a non-adaptive twodimensional grid in cylindrical coordinates with three levels of refinement. The given resolution $(\Delta r \times \Delta z)$ of Yorke \& Sonnhalter (2002) represents the case of a $M_{\text {core }}=60 \mathrm{M}_{\odot}$ pre-stellar core. The resolution for the lower mass $M_{\text {core }}=30 \mathrm{M}_{\odot}$ collapse was a factor of two better. The resolution for the higher mass $M_{\text {core }}=120 \mathrm{M}_{\odot}$ collapse was a factor of two worse. The simulations of Krumholz et al. (2007, 2009) were performed on a three-dimensional cartesian adaptive mesh refinement (AMR) grid. The given resolution $(\Delta x \times \Delta y \times \Delta z)$ represents the lowest and highest resolution during the simulation. The radiation from the sink cells is added to their computational grid using a smooth weighting function inside the so-called accretion radius, which is four times the highest resolution of the grid. (Krumholz 2005). The resolution of our own grids in spherical coordinates is given in units of arc length $(\Delta r \mathrm{x}(r \Delta \theta))$. 
outward direction. Thus, the choice of the floor value does not influence the level of accretion onto the newly forming star we investigate.

The various sources of additional acceleration $\vec{a}=\sum_{i} \vec{a}_{i}$ that enter the equations of hydrodynamics in Eqs. (3) and (4) are discussed in the following sections and include viscosity, gravity of the central star as well as self-gravity of the core, and radiative feedback.

\subsection{Viscosity}

In the two-dimensional simulations we consider physical shear viscosity of the circumstellar disk medium to mimic the effect of angular momentum transport (via e.g. the magneto-rotational instability, spiral arms, disk winds and jets). Two-dimensional axially symmetric simulations without any shear viscosity yield the formation of ring instabilities in the circumstellar disk. The rings would be unstable if non-axially symmetric modes were allowed, leading to the formation of spiral arms and therefore to angular momentum transport as discussed by Yorke et al. (1995).

Full physical tensor viscosity is included in the current version of the open source magnetohydrodynamics code Pluto3 (Mignone et al. 2007). Viscosity enters the equations of hydrodynamics in Eqs. (3) and (4) as an additional source of acceleration

$$
\vec{a}_{1}=\vec{\nabla} \Pi \text {. }
$$

The components of the viscous stress tensor $\Pi$ are given (in cartesian coordinates) by

$$
\Pi_{i j}=\eta\left(\partial_{j} u_{i}+\partial_{i} u_{j}-\frac{2}{3} \delta_{i j} \partial_{k} u_{k}\right)+\eta_{\mathrm{b}} \delta_{i j} \partial_{k} u_{k}
$$

with the shear viscosity $\eta$, the bulk viscosity $\eta_{\mathrm{b}}$, and the Kronecker symbol $\delta_{i j}$. Further details on the analytical treatment of viscosity can e.g. be found in Landau \& Lifshitz (1987). We assume for the bulk viscosity $\eta_{\mathrm{b}}=0$.

The (shear) viscosity

$$
\eta=\rho \nu
$$

is described via the so-called $\alpha$-parameterization of Shakura \& Sunyaev (1973), in which the dynamical viscosity $\nu$ is set proportional to the product of a typical velocity and length scale of the system under investigation, here the local sound speed $c_{\mathrm{s}}$ and pressure scale height $H$ :

$$
\nu=\alpha c_{\mathrm{S}} H
$$

We further approximate the local pressure scale height $H$ by

$$
H=\frac{c_{\mathrm{S}}}{\Omega_{\mathrm{K}}(r)}
$$

with the keplerian angular velocity

$$
\Omega_{\mathrm{K}}(r)=\sqrt{\frac{G M(r)}{r^{3}}}
$$


derived from the equilibrium between gravity and the centrifugal force. The mass $M(r)$ inside the radius $r$ is calculated by the spatial integral of the density distribution plus the central stellar mass $M_{*}$ inside the sink cell:

$$
M(r)=M_{*}+2 \pi \int_{0}^{r} d r \int_{0}^{\pi} d \theta \rho(r, \theta) r^{2} \sin (\theta) .
$$

Using the relation (10) we substitute the local sound speed in Eq. (9) yielding

$$
\nu=\alpha \Omega_{\mathrm{K}}(r) H^{2} .
$$

Introducing the dimensionless parameter $H / R$, the aspect ratio of the circumstellar disk, leads to

$$
\nu=\alpha \Omega_{\mathrm{K}}(r) R^{2}\left(\frac{H}{R}\right)^{2}
$$

with the cylindrical radius $R=r \sin (\theta)$. If the viscosity is an effect of turbulent transport of angular momentum, e.g. by the magneto-rotational instability Balbus \& Hawley (1991); Hawley \& Balbus (1991); Balbus (2003) or the baroclynic instability Klahr \& Bodenheimer (2003), it is observed that the strength of the stresses is proportional to the thermal pressure. This is the fundamental assumption of the $\alpha$-Ansatz by Shakura \& Sunyaev (1973). This relation (Eq. (9)) holds because hotter and thicker disks can support higher levels of turbulence. The situation is reversed for self-gravitating disks. Here, hot disks are usually Toomre stable and will not produce gravito-turbulence. On the contrary, the disks will cool down to the marginally unstable Toomre values and establish a turbulent state where the level of turbulence is set by the equilibrium of energy release and radiative cooling (Gammie 2001). For that reason, we choose a viscosity prescription independent on the actual disk temperature (e.g. a fixed $H / R$ ratio of 0.1 and $\alpha=0.3$ ) but only on the local mean (keplerian) rotation profile. This way, we ensure that cool and thin disks can obtain the high viscosity values they deserve. Our Ansatz is equivalent to the so-called $\beta$-viscosity Ansatz for self-gravitating disks by Duschl et al. (2000), which is also independent on temperature, with the $\beta$-parameter of $\beta \approx 3 * 10^{-3}$.

\subsection{Gravity}

The calculation of the gravitational potential $\Phi$ is split into the gravity of the central star in the sink cell $\Phi_{*}$ and the self-gravity of the mass in the computational domain $\Phi_{\mathrm{sg}}$ :

$$
\Phi=\Phi_{*}+\Phi_{\mathrm{sg}}
$$

The associated accelerations $\vec{a}_{2}+\vec{a}_{3}$ enter the hydrodynamics as a source term for momentum and energy in Eqs. (3) and (4).

The acceleration vector $\vec{a}_{2}$ of the gravity of the central star is given analytically by

$$
\vec{a}_{2}=-\vec{\nabla} \Phi_{*}=\vec{\nabla} \frac{G M_{*}}{r}=\partial_{r} \frac{G M_{*}}{r} \vec{e}_{r}=-\frac{G M_{*}}{r^{2}} \vec{e}_{r} .
$$


Such external gravity (from point sources) is supported in Pluto3 by defining the gravitational potential $\Phi_{*}$ or the resulting acceleration vector $\vec{a}_{2}$.

The acceleration $\vec{a}_{3}$ due to self-gravity is given by

$$
\vec{a}_{3}=-\vec{\nabla} \Phi_{\mathrm{sg}},
$$

in which the gravitational potential $\Phi_{\mathrm{sg}}$ is determined via Poisson's equation:

$$
\vec{\nabla}^{2} \Phi_{\mathrm{sg}}=4 \pi G \rho .
$$

We implemented a solver of Poisson's equation into our version of the Pluto code in a modular fashion. The module solves the equation via a diffusion ansatz. The desired approximate matrix inversion is done using the so-called GMRES method. The accuracy of the Poisson solver, i.e. the abort criterion for the approximate matrix inversion, is choosen to $0.001 \%$ relative accuracy of the gravitational potential $\left(\Delta \Phi_{\mathrm{sg}} / \Phi_{\mathrm{sg}} \leq 10^{-5}\right)$.

The outer radial boundary values of the gravitational potential are calculated via a Taylor expansion of the density distribution, described for example in Black \& Bodenheimer (1975). Several tests we performed indicate that it is sufficient to just account for the monopole solution of the Taylor expansion, i.e. the total mass of the core. In our default configuration of the pre-stellar cores (see Sect. 3), the mass distribution is perfectly spherically symmetric at the beginning of the simulation and afterwards becomes highly concentrated in the inner region of the computational domain far away from the outer boundary, both yield analytically the monopole solution at the outer boundary. To control the resolution, which is necessary to resolve the physics of self-gravity correctly, e.g. preventing artificial fragmentation, we monitor the so-called Truelove criterion, derived in Truelove et al. (1997). The criterion requires to resolve the Jeans length

$$
\lambda_{\mathrm{J}}=\sqrt{\frac{\pi c_{\mathrm{s}}^{2}}{G \rho}}
$$

at least by a priorily defined number of grid cells. The inverse of the number of necessary grid cells per Jeans length is the so-called Jeans number

$$
J=\frac{\Delta x}{\lambda_{\mathrm{J}}}
$$

Truelove et al. (1997) suggested at least a Jeans number of $J \leq 0.25$. The worst case during our simulations occurs in the high-density region around the forming star with approximately a maximum density of $\rho \leq 10^{-11} \mathrm{~g} \mathrm{~cm}^{-3}$, a minimum temperature of $T \approx 100 \mathrm{~K}$ and a resolution of the order of $\Delta r \approx 1 \mathrm{AU}$. This leads to a Jeans number of $J \approx 0.09$, i.e. the Jeans length $\lambda_{\mathrm{J}}$ is at least resolved by 11 grid cells.

\subsection{Radiation transport}

The importance of the frequency dependence of the stellar spectrum when calculating the radiative feedback of a massive star was already shown in radiation hydrodynamics studies by 
Yorke \& Sonnhalter (2002) and Edgar \& Clarke (2003) as well as in radiation transport simulations without hydrodynamics by Krumholz et al. (2005). On the other hand, no frequency dependent radiation hydrodynamics study related to massive star formation was carried out since the work by Yorke \& Sonnhalter (2002) in more than one dimension and due to the huge computational overhead of their frequency dependent FLD routine it was neither possible to study a large number of different initial conditions (to scan the parameter space), nor to perform high-resolution simulations of the accretion process.

Furthermore, stellar radiative feedback onto the dynamics of the environment plays a crucial role in the formation of massive stars. On the one hand, the heating will probably prevent further fragmentation of the cloud by enhancing the Jeans mass (e.g. Krumholz et al. 2007). On the other hand, the dusty environment feels the radiative force when absorbing the radiation due to momentum conservation, which potentially stops the accretion process for highly luminous massive stars.

To study the radiative feedback of massive stars on their own accretion stream in one-, two-, and three-dimensional simulations we implemented a fast, robust, and accurate frequency dependent radiation transport solver in spherical coordinates into our version of the Pluto code. To achieve a fast solver for the frequency dependent problem we split the radiation field into the stellar irradiation and thermal dust emission. The basic methodology of the hybrid scheme is to perform a frequency dependent ray-tracing step for the stellar irradiation and shift the re-emission of the photons by the dusty environment to a frequency averaged flux limited diffusion solver in the equilibrium temperature approximation. A derivation of the hybrid scheme and numerical details of the implementation of this newly developed radiation transport method are given in Kuiper et al. (2010), including a detailed comparison of the method with the standard radiation transport benchmark test by Pascucci et al. (2004).

\subsection{Dust model}

For the implementation of realistic mass absorption coefficients $\kappa(\nu)$ for the frequency dependent radiation transport module, we use an opacity table of Laor \& Draine (1993), including 79 frequency bins, shown in Fig. 2. The opacity table covers the full frequency range from microwave

and infrared radiation up to soft x-rays. It describes a mixture of dust grains in the size range between 0.005 to $10.0 \mu \mathrm{m}$. The grains are taken to be spherical and consist out of amorphous silicate with a composition like that of olivine. The model does not include ice mantles, which will roughly double the opacity at temperatures below $\sim 100 \mathrm{~K}$ (e.g. Ossenkopf \& Henning 1994). As shown in Fig. 2 this dust grain mixture takes into account the strong absorption/emission features at $9.7 \mu \mathrm{m}$ and $18 \mu \mathrm{m}$ observed in the interstellar medium. The corresponding frequency averaged Planck- and Rosseland mean opacities are shown in Fig. 3 as a function of temperature.

Aside from the dust opacities, the opacity of a given grid cell depends also linearly on the local 


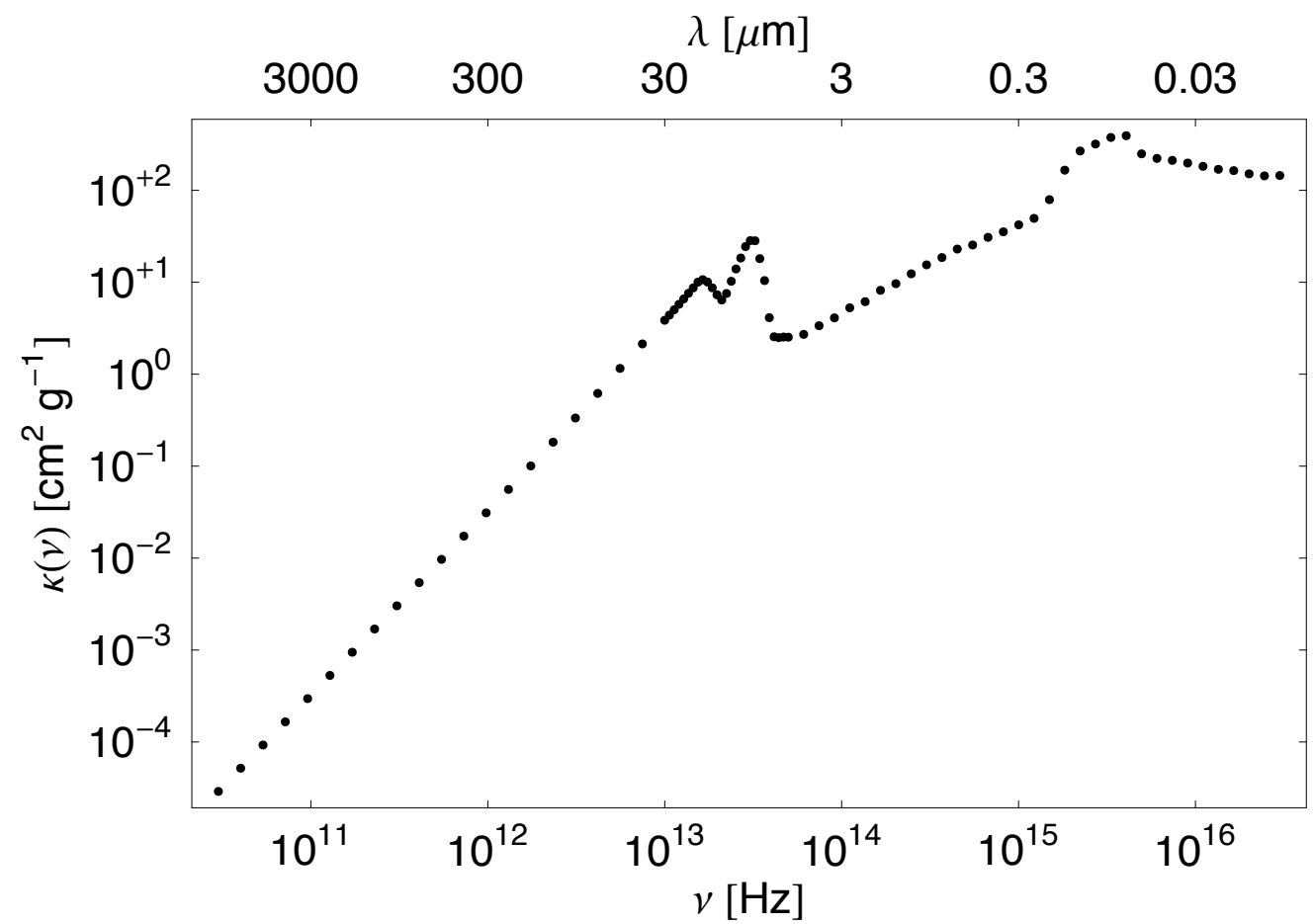

Fig. 2.- : Frequency dependent mass absorption coefficients $\kappa(\nu)$ in tabulated form from Laor \& Draine (1993). 


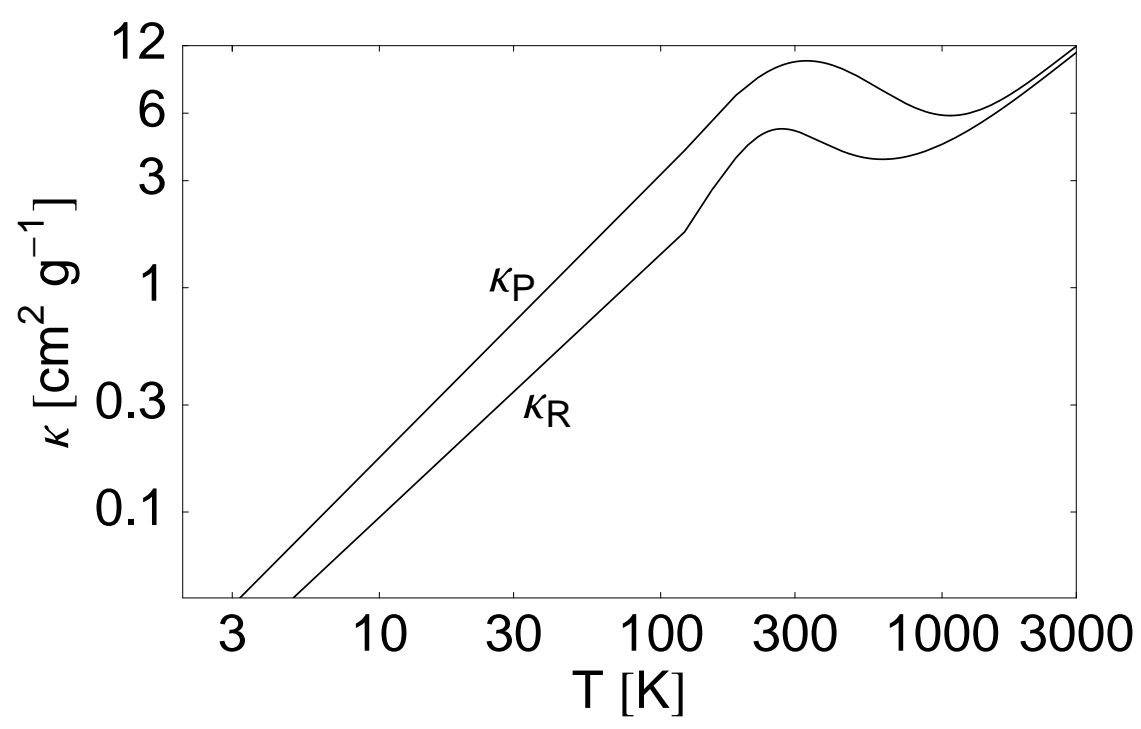

Fig. 3.-: Calculated Rosseland $\kappa_{\mathrm{R}}$ and Planck $\kappa_{\mathrm{P}}$ mean opacities as a function of dust temperature. Here, the possible evaporation of dust grains at high temperatures (and/or low densities) is neglected, but considered in the dust to gas mass ratio $M_{\text {dust }} / M_{\text {gas }}$ of each grid cell, cp. Fig. 4 .

dust to gas mass ratio. The initial dust to gas mass ratio $\left(M_{\text {dust }} / M_{\text {gas }}\right)_{0}$ is fixed to $1 \%$. Gas and dust is treated as a single fluid, so the dust to gas mass ratio only shrinks due to possible evaporation of the dust grains in hot regions (around the central massive star). The local evaporation temperature of the dust grains is calculated by using the formula of Isella \& Natta (2005)

$$
T_{\text {evap }}=g \rho^{\beta}
$$

with $g=2000 \mathrm{~K}, \beta=0.0195$, and the gas density $\rho$ given in $\mathrm{g} \mathrm{cm}^{-3}$. The formula describes a power-law approximation to the evaporation temperatures $T_{\text {evap }}$ determined by Pollack et al. (1994). A smooth spatial and temporal transition of the associated dust to gas mass ratio between completely evaporated and condensated regions is achieved via the transition function

$$
\frac{M_{\text {dust }}}{M_{\text {gas }}}(\vec{x})=\left(\frac{M_{\text {dust }}}{M_{\text {gas }}}\right)_{0}\left(0.5-\frac{1}{\pi} \arctan \left(\frac{T(\vec{x})-T_{\text {evap }}(\vec{x})}{100}\right)\right) .
$$

The transition slope is displayed in Fig. 4 as a function of the temperature for a high gas density of $\rho=10^{-10} \mathrm{~g} \mathrm{~cm}^{-3}$ as well as for the floor value of the density $\rho_{0}=10^{-21} \mathrm{~g} \mathrm{~cm}^{-3}$. 


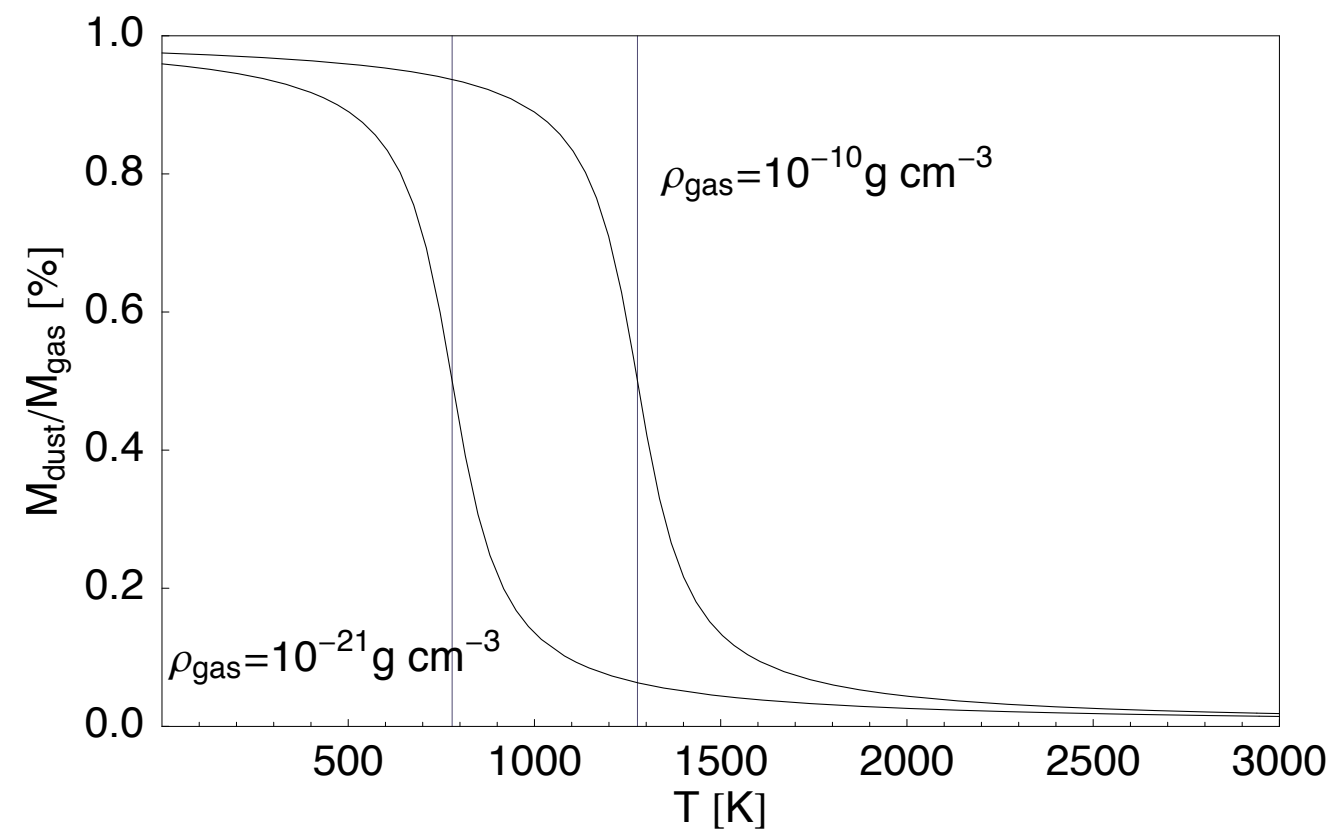

Fig. 4.- : Transition slope of the local dust to gas mass ratio as a function of the temperature due to evaporation of dust grains for two different gas densities. The vertical lines mark the corresponding evaporation temperatures.

\subsection{Stellar evolution model}

The evolution of the central star, described by a central sink cell, is coupled to the hydrodynamics of the pre-stellar core by measuring the mass flux into the sink cell. The initial mass of the star at the beginning of the simulation is simply given by the integral over the initial density distribution up to the radius $r_{\min }$ of the sink cell and is therefore in all cases less than a few percent of $1 \mathrm{M}_{\odot}$. The mass, which enters the sink cell during the hydrodynamics is assumed to be accreted onto the central star. From the mass flux $\rho \vec{u}$ into the sink cell during the timestep $\Delta t$ we calculate the accretion rate $\dot{M}$ onto the central star via

$$
\dot{M}=2 \pi \int_{0}^{\pi} d \theta \rho \vec{u} \cdot \vec{e}_{r} r_{\min }^{2} \sin \theta .
$$

Integrating the accretion rate $\dot{M}$ over the time yields the growth of the stellar mass $M_{*}$ :

$$
M_{*}(t+\Delta t)=M_{*}(t)+\int_{t}^{t+\Delta t} \dot{M} d t=M_{*}(t)+\dot{M} \Delta t .
$$

A potential decrease of the accretion rate due to outflows or jets (driven by magnetic forces) in the inner region below the sink cell radius, is currently not considered. The total luminosity $L_{\text {tot }}$ is given by the sum of the accretion luminosity $L_{\text {acc }}$ and the stellar luminosity $L_{*}$ :

$$
L_{\mathrm{tot}}(t)=L_{\mathrm{acc}}(t)+L_{*}(t)
$$


The accretion luminosity is directly calculated from the hydrodynamics simulation via

$$
L_{\mathrm{acc}}=\frac{G M_{*}}{R_{*}} \dot{M}
$$

with the stellar radius $R_{*}$. The stellar luminosity and the stellar radius are obtained via fits to the pre-calculated evolutionary tracks by Hosokawa \& Omukai (2009). These evolutionary tracks of massive stars depend on the stellar mass as well as on the actual accretion rate. We use polynomial fits to the mass relation up to $10^{\text {th }}$ order for separated mass ranges (an example of these fits is shown in Fig. 5) and linear regression for the dependency on the instantaneous accretion rate. For stellar masses below the accessible data $\left(0.05 \mathrm{M}_{\odot}\right.$ in the worst case) the stellar luminosity is

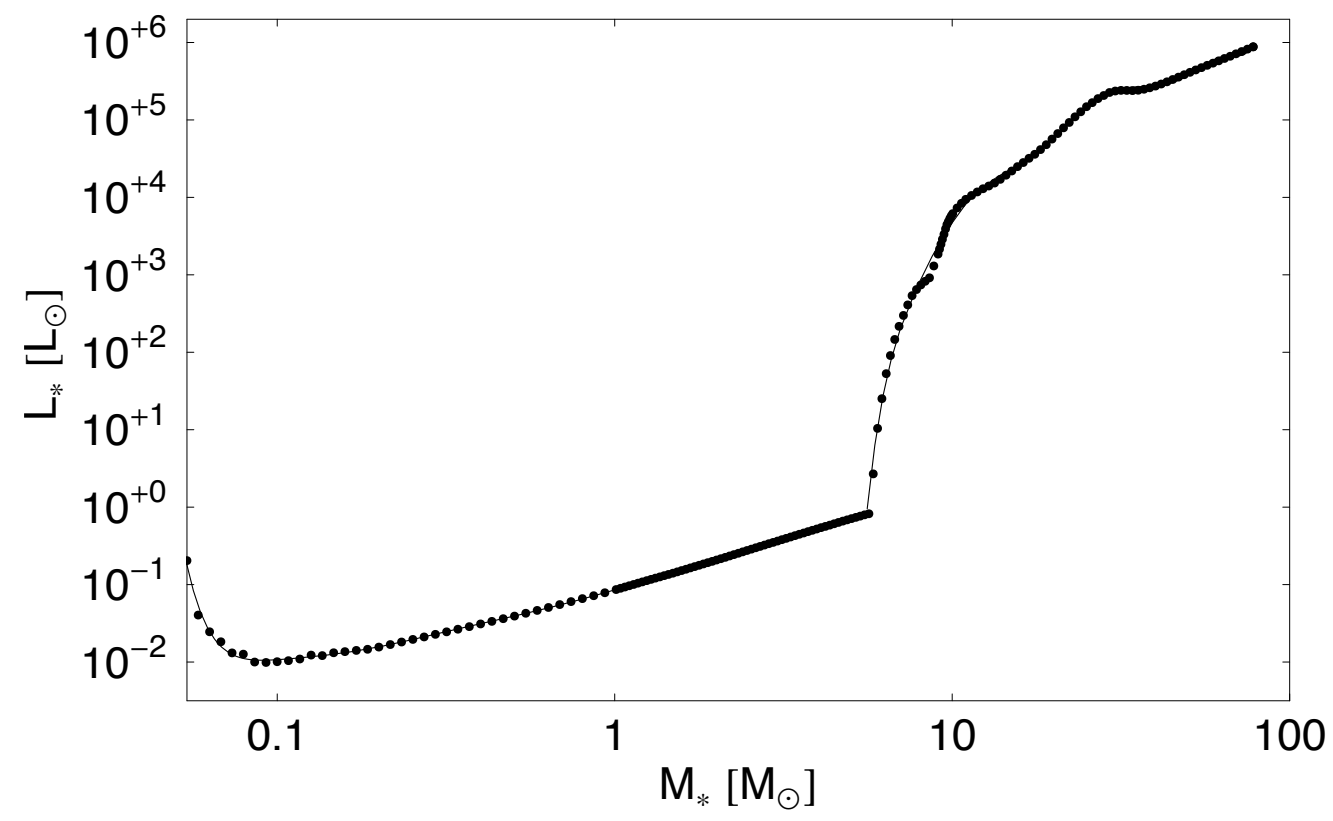

Fig. 5.- : Polynomial fits to the stellar luminosity as a function of the stellar mass as calculated by Hosokawa \& Omukai (2009). The data points represent an evolving massive star with an accretion rate of $10^{-3} \mathrm{M}_{\odot} \mathrm{yr}^{-1}$. The mass range was split into two regimes above and below $5.5 \mathrm{M}_{\odot}$ (at the sharp bend) and each part is fitted by a polynomial up to $10^{\text {th }}$ order (solid lines).

assumed to be negligible and the stellar radius is assumed to be constant up to the first data point.

Given the stellar radius and total luminosity, the stellar effective temperature $T_{*}$ is calculated from

$$
L_{\mathrm{tot}}=4 \pi R_{*}^{2} \sigma_{\mathrm{SB}} T_{*}^{4} .
$$




\section{Initial conditions and numerical configuration}

Using the newly developed modules of the self-gravity radiation hydrodynamics code presented in Sect. 2, we performed multiple simulations of collapsing massive pre-stellar cores. Most of the simulations were performed either to scan the huge numerical parameter space of the setup to guarantee significant results or to explore individual physical initial conditions. An overview of the 22 simulations evaluated is presented in Table 2 and Table 3 for one- and two-dimensional simulations respectively.

Aside from varying one specific parameter of the initial condition or the numerical configuration in each simulation series, most of the initial conditions and the physics considered in the simulations stay the same.

Our basic initial condition is very similar to the one used by Yorke \& Sonnhalter (2002). We start from a cold $\left(T_{0}=20 \mathrm{~K}\right)$ pre-stellar core of gas and dust. The initial dust to gas mass ratio is chosen to be $M_{\text {dust }} / M_{\text {gas }}=1 \%$. The model describes a so-called quiescent collapse scenario without turbulent motion $\left(\vec{u}_{r}=\vec{u}_{\theta}=0\right)$. In non-spherically symmetric two-dimensional runs the core is initially in slow rigid rotation $\left(\left|\vec{u}_{\phi}\right| / R=\Omega_{0}=5 * 10^{-13} \mathrm{~Hz}\right)$. The rotation speed of $\Omega_{0}$ results roughly in an equilibrium between gravity and centrifugal force at the outer core radius $r_{\max }$ in the case of the lowest mass core of $M_{\text {core }}=60 \mathrm{M}_{\odot}$. The outer radius of the cores is fixed to $r_{\max }=0.1 \mathrm{pc}$ and the total mass $M_{\text {core }}$ varies in the simulations from 60 up to $480 \mathrm{M}_{\odot}$. The initial density slope drops with $r^{-2}$. The highest mass case of $480 \mathrm{M}_{\odot}$ with a mean density of $\bar{\rho} \approx 8 * 10^{-18} \mathrm{~g} \mathrm{~cm}^{-3}\left(\approx 2 * 10^{6} \mathrm{~cm}^{-3}\right)$ denotes the upper mass limit of such a pre-stellar core we would expect from observations. A brief overview of these physical initial conditions of the massive pre-stellar cores studied is given in Table 4 .

The simulations are performed on a time independent grid in spherical coordinates (see Sect. 2.1). The radially inner boundary of the computational domain is a semi-permeable wall towards the forming star, i.e. the gas can enter the central sink cell, but it cannot leave. The outer radial boundary is a semi-permeable wall as well. The mass can be pushed out of the computational domain (by radiative forces) but no mass is allowed to enter the computational domain. The semipermeable outer boundary implies the assumption that the collapsing core is mostly isolated from its large-scale environment. This limits the extent of the potential mass reservoir for the forming massive star to the initially fixed mass of the pre-stellar core $M_{\text {core }}$.

The remaining numerical parameters are determined in several simulation series. The resolution of the computational domain, which is necessary to follow the radiation and fluid physics as well as its interactions, is determined in several so-called convergence runs, see Sects. A.1 and A.2 for non-rotating and rotating cores respectively. The highest resolution of the non-uniform grid is chosen around the forming massive star, afterwards the resolution decreases logarithmically in the radial outward direction. The default resolution goes down to $(\Delta r)_{\min }=0.08 \mathrm{AU}$ and $(\Delta r \times r \Delta \theta)_{\min }=1.27 \mathrm{AU} \times 1.04 \mathrm{AU}$ for the one- and two-dimensional simulations respectively. The accurate size of the sink cell is determined in parameter scans presented in Sects. 4.1 and 5.1 for 
Table 2. Overview of spherically symmetric massive pre-stellar core collapse simulations

\begin{tabular}{|c|c|c|c|c|c|c|c|}
\hline Label & Dim. & Grid cells & $\begin{array}{c}\text { Resol. } \\
(\mathrm{AU})\end{array}$ & $\begin{array}{l}r_{\min } \\
(\mathrm{AU})\end{array}$ & $\begin{array}{l}M_{\text {core }} \\
\left(\mathrm{M}_{\odot}\right)\end{array}$ & $\begin{array}{c}t_{\mathrm{ff}} \\
(\mathrm{kyr})\end{array}$ & $\begin{array}{c}t_{\text {end }} \\
(\mathrm{kyr})\end{array}$ \\
\hline 1D Convergence runs & & Sect. A.1 & & & & & \\
\hline 1D-Convergence32 & $1 \mathrm{D}$ & 32 & 0.36 & 1 & 60 & 67.6 & $310^{* *}$ \\
\hline 1D-Convergence64 & $1 \mathrm{D}$ & 64 & 0.17 & 1 & 60 & 67.6 & $163^{* *}$ \\
\hline 1D-Convergence128 & \multicolumn{7}{|c|}{ see '1D-Mcore60Msol' } \\
\hline 1D-Convergence256 & $1 \mathrm{D}$ & 256 & 0.04 & 1 & 60 & 67.6 & $188^{*}$ \\
\hline 1D rmin runs & & Sect. 4.1 & & & & & \\
\hline 1D-rmin1AU & $1 \mathrm{D}$ & $99+128$ & 1.0 & 1 & 60 & 67.6 & $204^{*}$ \\
\hline 1D-rmin5AU & $1 \mathrm{D}$ & $95+128$ & 1.0 & 5 & 60 & 67.6 & $282^{* *}$ \\
\hline 1D-rmin10AU & $1 \mathrm{D}$ & $90+128$ & 1.0 & 10 & 60 & 67.6 & $283^{* *}$ \\
\hline 1D-rmin $80 \mathrm{AU}$ & $1 \mathrm{D}$ & $20+128$ & 1.0 & 80 & 60 & 67.6 & $293^{*}$ \\
\hline 1D Mcore runs & & Sect. 4.2 & & & & & \\
\hline 1D-Mcore60Msol & $1 \mathrm{D}$ & 128 & 0.08 & 1 & 60 & 67.6 & $218^{* *}$ \\
\hline 1D-Mcore120Msol & $1 \mathrm{D}$ & 128 & 0.08 & 1 & 120 & 47.8 & $54^{* *}$ \\
\hline 1D-Mcore240Msol & $1 \mathrm{D}$ & 128 & 0.08 & 1 & 240 & 33.8 & $39^{* *}$ \\
\hline 1D-Mcore480Msol & $1 \mathrm{D}$ & 128 & 0.08 & 1 & 480 & 23.9 & $10^{* *}$ \\
\hline
\end{tabular}

Note. - The table is structured in blocks of topics and their corresponding sections. For each run the label, the dimension, the number of grid cells, the resolution of the best resolved region around the central star, the radius $r_{\text {min }}$ of the central sink cell, the initial mass $M_{\text {core }}$ of the pre-stellar core, its corresponding free fall time $t_{\mathrm{ff}}=\pi r_{\max }^{3 / 2} / \sqrt{8 G M_{\text {core }}}$, and the period $t_{\text {end }}$ of evolution simulated are given. A ${ }^{*}$ ' in the $t_{\text {end }}$ column denotes that the whole accretion phase of the star has been computed. A ${ }^{* *}$ ' denotes that the computation has been stopped at the point in time when no mass is left in the computational domain. 
Table 3. Overview of axially symmetric massive pre-stellar core collapse simulations

\begin{tabular}{|c|c|c|c|c|c|c|c|}
\hline Label & Dim. & Grid cells & $\begin{array}{c}\text { Resol. } \\
(\mathrm{AU})\end{array}$ & $\begin{array}{l}r_{\min } \\
(\mathrm{AU})\end{array}$ & $\begin{array}{l}M_{\text {core }} \\
\left(\mathrm{M}_{\odot}\right)\end{array}$ & $\begin{array}{c}t_{\mathrm{ff}} \\
(\mathrm{kyr})\end{array}$ & $\begin{array}{c}t_{\text {end }} \\
(\mathrm{kyr})\end{array}$ \\
\hline 2D Convergence runs & \multicolumn{7}{|c|}{ Sect. A.2 } \\
\hline 2D-Convergence $32 \mathrm{x} 16$ & $2 \mathrm{D}$ & $32 \times 16$ & $2.69 \times 1.11$ & 10 & 60 & 67.6 & 62 \\
\hline 2D-Convergence64x4 & $2 \mathrm{D}$ & $64 \times 4$ & $1.27 \times 4.18$ & 10 & 60 & 67.6 & 93 \\
\hline 2D-Convergence64x8 & $2 \mathrm{D}$ & $64 \times 8$ & $1.27 \times 2.09$ & 10 & 60 & 67.6 & $691^{* *}$ \\
\hline 2D-Convergence64x16 & \multicolumn{7}{|c|}{ see '2D-Mcore60Msol' } \\
\hline 2D-Convergence128x32 & $2 \mathrm{D}$ & $128 \times 32$ & $0.61 \times 0.51$ & 10 & 60 & 67.6 & $33^{+}$ \\
\hline $2 \mathrm{D}$ rmin runs & \multicolumn{7}{|c|}{ Sect. 5.1} \\
\hline 2D-rmin80AU & $2 \mathrm{D}$ & $64 \times 16$ & $7.25 \times 8.21$ & 80 & 60 & 67.6 & $251^{*}$ \\
\hline 2D-rmin10AU & \multicolumn{7}{|c|}{ see '2D-Mcore60Msol' } \\
\hline 2D-rmin5AU & $2 \mathrm{D}$ & $64 \times 16$ & $0.69 \times 0.52$ & 5 & 60 & 67.6 & $631^{+}$ \\
\hline 2D-rmin1AU & $2 \mathrm{D}$ & $64 \times 16$ & $0.17 \times 0.11$ & 1 & 60 & 67.6 & $92^{+}$ \\
\hline 2D Mcore runs & \multicolumn{7}{|c|}{ Sect. 5.2} \\
\hline 2D-Mcore60Msol & $2 \mathrm{D}$ & $64 \times 16$ & $1.27 \times 1.04$ & 10 & 60 & 67.6 & $939^{* *}$ \\
\hline 2D-Mcore120Msol & $2 \mathrm{D}$ & $64 \times 16$ & $1.27 \times 1.04$ & 10 & 120 & 47.8 & $489^{* *}$ \\
\hline 2D-Mcore240Msol & $2 \mathrm{D}$ & $64 \times 16$ & $1.27 \times 1.04$ & 10 & 240 & 33.8 & $226^{* *}$ \\
\hline 2D-Mcore480Msol & $2 \mathrm{D}$ & $64 \times 16$ & $1.27 \times 1.04$ & 10 & 480 & 23.9 & $41^{+}$ \\
\hline
\end{tabular}

Note. - The table is structured in the same way as Table 2. Simulations, which are still running, are marked by an additional ' + ' in the $t_{\text {end }}$ column. 
non-rotating and rotating cores respectively. While we started the first two-dimensional simulations with a radius of the inner sink cell of $r_{\text {min }}=80 \mathrm{AU}$ analog to the case of 'F60' in Yorke \& Sonnhalter (2002) we experienced that it is necessary to shrink the size of the sink cell down to a value smaller than the distance from the dust sublimation front to the forming massive star, at least from the point in time on at which the radiative force becomes a serious counterpart to the gravity. Otherwise a huge sink cell give rise to an artificially high radiative feedback and therefore limits the stellar mass reached in the simulations dramatically. The default radius of the sink cell is chosen to be $r_{\min }=1 \mathrm{AU}$ and $r_{\min }=10 \mathrm{AU}$ for the one- and two-dimensional simulations respectively. In axially symmetric (two-dimensional) runs, physical shear viscosity is used to maintain the accretion flow through the growing circumstellar disk. Therefore, we adopted the well-known $\alpha$-parametrization model for shear viscosity of standard disk theory (Shakura \& Sunyaev 1973). We performed several simulations with varying normalization values for the physical $\alpha$-viscosity, which yield the formation of a stable accretion disk for a range of $\alpha$-values from 0.1 up to 1.0. Apart from these runs the normalization of the viscosity was fixed to be $\alpha=0.3$ here. A theoretical estimation on the $\alpha$-values of massive accretion disks was presented in Vaidya et al. (2009).

In previous test runs, we studied several non-radiative and radiative physics. We performed isothermal and adiabatic collapse simulations as well as gray and frequency dependent radiation transport with and without radiation pressure feedback from the star or the diffuse thermal radiation field. In this paper, we confine ourselves to present only the most realistic runs including frequency dependent radiation transport as well as full radiative feedback.

\section{Spherically symmetric accretion}

As mentioned in the introduction, spherically symmetric accretion onto a massive star is potentially stopped by its growing radiation pressure. First, we present our results of one-dimensional simulations used to fix the numerical parameters of the setup, namely, the resolution of the computational grid (see Sect. A.1) and the radius of the inner sink cell (Sect. 4.1). Afterwards, we analyze simulations with varying initial core masses $M_{\text {core }}$ to determine the upper mass limit, if such a limit exists, for our specific model (the chosen dust and stellar evolution model, the configuration of the hydrodynamics solver as well as the treatment of radiation transport). To re-perform these onedimensional simulations on our own instead of just referring to Larson \& Starrfield (1971), Kahn (1974), Yorke \& Krügel (1977), and Wolfire \& Cassinelli (1987) allows us to directly compare the results found for spherically symmetric accretion flows with subsequent simulation results in higher dimensions. 


\subsection{Parameter scan of the size of the sink cell: The influence of the dust sublimation front}

\subsubsection{Simulations}

In order to limit the run time of the simulations to an adequate amount, the formation and evolution of the central proto-star cannot be included in the computational domain. In fact, the radially inner computational boundary defines the radius of a so-called sink cell. The mass flux into this sink cell defines the accretion rate onto the proto-star, which is assumed to form in the center of the pre-stellar core. Inside of this sink cell the stellar properties such as luminosity and radius are taken from pre-calculated stellar evolutionary tracks. We use therefore recent results for the evolution of accreting high-mass stars by Hosokawa \& Omukai (2009). In the following, we study the influence of the location $r_{\min }$ of this inner boundary on the resulting accretion rate onto the evolving massive star. We check this dependency in four simulations with a radius of the inner sink cell of $r_{\min }=1,5,10$, and $80 \mathrm{AU}$. To decouple the results from the dependence on resolution (see Sect. A.1) the size of the grid cells was fixed to be $\Delta r=1$ AU up to a radius of 100 AU. So the different simulations use 99, 95, 90, and 20 grid cells up to 100 AU respectively. Behind this inner region, the grid resolution decreases logarithmically throughout additional 128 grid cells from 100 AU up to $0.1 \mathrm{pc}$. The initial conditions and numerical parameters of these runs are described in Sect. 3 and the simulations are performed for an initial core mass of $M_{\text {core }}=60 \mathrm{M}_{\odot}$. We follow the long-term evolution of the runs for at least $200 \mathrm{kyr}$, representing 3.0 free fall times. The resulting accretion flow onto the forming star as well as the deviations of the simulations from the run with the smallest sink cell $r_{\min }=1 \mathrm{AU}$ are displayed in Fig. 6 .

\subsubsection{Conclusions}

The first absorption of stellar irradiation takes place directly behind the dust sublimation radius $r_{\text {subl }}$. If the radius of the central sink cell $r_{\text {min }}$ exceeds this dust sublimation radius, this interaction is artificially shifted to $r_{\min }$. Due to the fact that the generalized Eddington limit is independent of the radius (the stellar gravity and the stellar radiative flux both drop with $r^{-2}$ ), the shift of this first transfer of momentum from the stellar irradiation to the dust flow should be independent of the radius of the sink cell. Secondly, the absorption of stellar irradiation heats up the region behind the dust sublimation radius respectively. The thermal radiative flux from this region outwards slows down the gravitationally in-falling accretion flow. In general, this interaction depends on the radius, which defines the temperature of the heated region, the velocity of the accretion flow and the opacity of the corresponding dust.

In the plot of the resulting accretion rates (Fig. 6, upper panel) only slight deviations of the run with the largest sink cell radius $r_{\min }=80 \mathrm{AU}$ are visible during the initial and final epoch. The

other runs show identical results. The lower panel of Fig. 6 shows in more detail the deviations of 

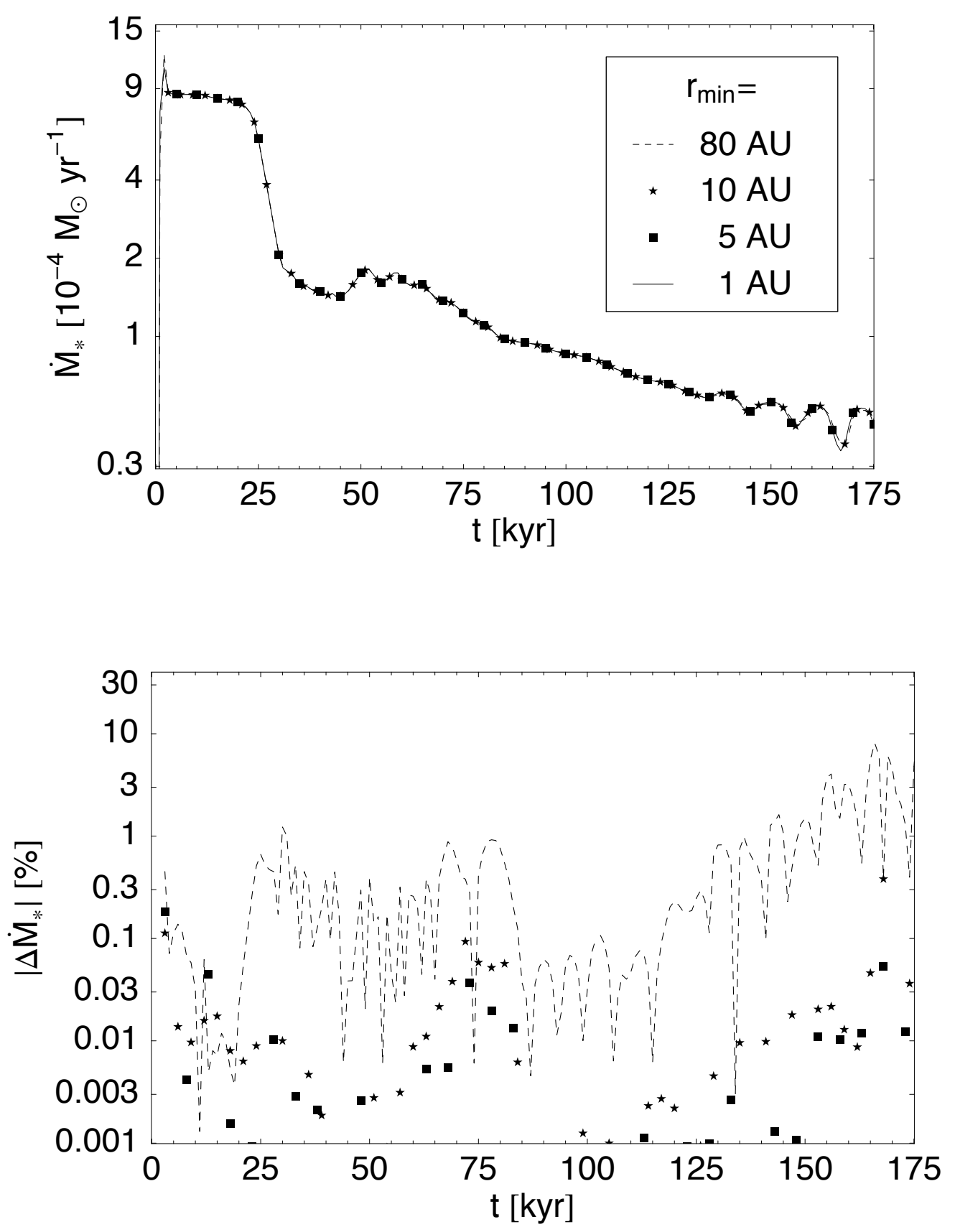

Fig. 6.- : Accretion rate (upper panel) and deviations of the accretion rates from the simulation run with the smallest sink cell radius of $r_{\min }=1 \mathrm{AU}$ (lower panel) as a function of time for four different sizes of the spherical sink cell. 
the simulations from the run with $r_{\min }=1 \mathrm{AU}$. The simulation runs with $r_{\min }=10,5$, and $1 \mathrm{AU}$ stay identical. In these simulations the dust sublimation radius, which can be roughly estimated to 20 to $30 \mathrm{AU}$ for a corresponding 20 to $30 \mathrm{M}_{\odot}$ star, is included in the computational domain before the onset of radiation pressure occurs at roughly $25 \mathrm{kyr}$. On the other hand, the largest sink cell of $r_{\min }=80 \mathrm{AU}$ exceeds the dust sublimation radius $r_{\text {subl }}$. The resulting accretion rate of the corresponding run oscillates around the results from the more precise simulations with a maximum deviation of $10 \%$ mostly at the end of the simulation, when the radiation pressure starts to revert the accretion flow throughout the whole domain. Due to the fact that the deviations are oscillating and the fact that the strongest deviations occur at the end of the simulation where the accretion rate is already an order of magnitude lower than at the beginning, the four simulations yield the same final mass of the proto-star. Subsequent one-dimensional simulations presented make use of a radius of the central sink cell of $r_{\min }=1 \mathrm{AU}$.

\subsection{Parameter scan of the initial pre-stellar core mass: The upper mass limit of spherically symmetric accretion}

\subsubsection{Simulations}

The simulations presented so far were performed to fix the remaining free numerical parameters, namely, the grid resolution and the size of the central sink cell. We now study the collapse of massive pre-stellar cores for four different initial core masses $M_{\text {core }}$ ranging from $M_{\text {core }}=60 \mathrm{M}_{\odot}$ up to $480 \mathrm{M}_{\odot}$. The initial conditions and numerical parameters for these runs are described in

Sect. 3. The simulations are performed with an inner boundary of the computational domain of $r_{\min }=1 \mathrm{AU}$ and 128 grid cells with logarithmically increasing resolution towards the center. The size of the innermost grid cell of the computational domain is $(\Delta r)_{\min }=0.08 \mathrm{AU}$. We follow the evolution of the system until no mass is left in the computational domain. Part of this mass is accreted onto the central massive star and part is expelled over the outer boundary by radiative forces. The resulting accretion histories are displayed in Fig. 7 as a function of the actual stellar mass.

\subsubsection{Conclusions}

The mass and the luminosity of the forming massive star grow with time. The radiation pressure of the direct stellar irradiation as well as from the thermal infrared dust emission increase and become stronger than gravity ultimately. Therefore, the accretion rate drops down and the massive star has grown to its final mass.

The individual force densities as as function of the radius through the spherically symmetric pre-stellar core are displayed at a snapshot in time, where the radiative force starts to trigger the 
Table 4. Overview of initial conditions

\begin{tabular}{ccl}
\hline \hline Symbol & Value & \multicolumn{1}{c}{ Quantity } \\
\hline$T_{0}$ & $20 \mathrm{~K}$ & temperature of the pre-stellar core \\
$\left(M_{\text {dust }} / M_{\text {gas }}\right)_{0}$ & $1 \%$ & dust to gas mass ratio \\
$\left|\vec{u}_{r}\right|$ & 0 & radial velocity \\
$\left|\vec{u}_{\theta}\right|$ & 0 & polar velocity \\
$\Omega_{0}=\left|\vec{u}_{\phi}\right| / R$ & $5 * 10^{-13} \mathrm{~Hz}$ & azimuthal angular velocity in 2D \\
$r_{\max }$ & $0.1 \mathrm{pc}$ & outer radius of the pre-stellar core \\
$\rho(r)$ & $r^{-2}$ & density slope of the pre-stellar core \\
$M_{\text {core }}$ & 60 to $480 \mathrm{M}_{\odot}$ & mass of the pre-stellar core \\
\hline
\end{tabular}

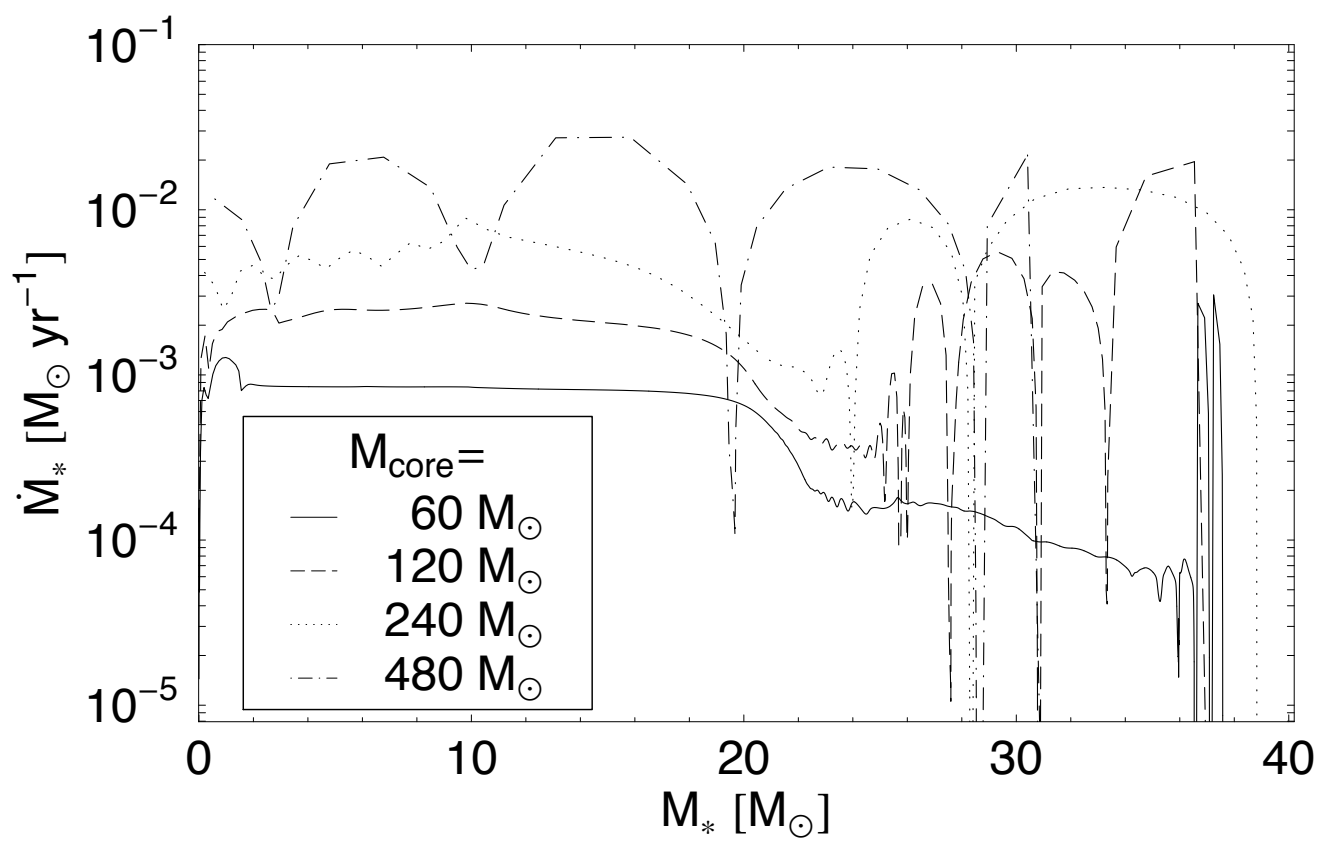

Fig. 7.-: Accretion rate $\dot{M}_{*}$ as a function of the actual stellar mass $M_{*}$ for four different initial pre-stellar core masses of $M_{\text {core }}=60 \mathrm{M}_{\odot}$ up to $480 \mathrm{M}_{\odot}$. The spherically symmetric accretion models yield an upper mass limit of the final star of $M_{*}^{1 \mathrm{D}}<40 \mathrm{M}_{\odot}$. 
stopping of the in-fall motion in Fig. 8. These forces are later on compared with the corresponding forces of the disk accretion models.

The final star does not reach a mass higher than $40 \mathrm{M}_{\odot}$ in any of the simulations. This limit is in good agreement with previous research studies. It lies in the allowed range of 25 $60 \mathrm{M}_{\odot}$ determined by Larson \& Starrfield (1971). Kahn (1974) predicted in his analytical study the formation of a $40 \mathrm{M}_{\odot}$ star and Yorke \& Krügel (1977) formed a $36 \mathrm{M}_{\odot}$ star in their radiation hydrodynamics simulation of a $150 \mathrm{M}_{\odot}$ collapsing core.

The oscillations of the accretion rate during the stopping of the in-fall motion are due to a negative feedback effect of the accretion luminosity: By increasing the initial mass of the prestellar core from $60 \mathrm{M}_{\odot}$ up to $480 \mathrm{M}_{\odot}$, the amplitude of the accretion rate and therefore the accretion luminosity increases as well. Due to the resulting stronger radiative force, the increase of accretion luminosity leads to a de-acceleration of the accretion flow, which results in a reduction of the corresponding accretion luminosity. This negative feedback yields a highly episodic accretion history. The effect is stronger in cases, where the ratio of the accretion luminosity to the stellar luminosity is high, i.e. the effect is stronger for more massive cores. Such an oscillating phase was also previously detected in the simulations by Yorke \& Krügel (1977).

The fact that the final mass of the star in the most massive case $M_{\text {core }}=480 \mathrm{M}_{\odot}$ is lower $\left(M_{*} \approx\right.$ $31 \mathrm{M}_{\odot}$ ) than for the cores that initially had less mass, should be taken with care: In simulations with such high oscillations, the influence of the underlying stellar evolution model increases strongly. To analyze the details of this time dependent interaction of the stellar evolution and the accretion flow, a self-consistent treatment of the proto-stellar's evolution and its environment should be considered.

\section{Disk accretion}

The most massive stars known cannot be formed by spherically symmetric accretion. As shown in the last section, the radiative forces in a spherically symmetric envelope lead to a cut-off of the accretion phase. The high luminosity of a massive star heats the region in its vicinity to such a high temperature that the resulting thermal radiation pressure overcomes the gravitational force. The radiation pressure stops, and finally reverses the accretion flow. Besides this theoretical issue, observations indicate the presence of angular momentum in all epochs of star formation, starting with the rotation of pre-stellar cores and finally resulting in rotating flattened circumstellar structures. Leaving perfectly spherical symmetry will thereby potentially help to overcome the radiation pressure problem. First, the presence of higher densities in the forming disk region results in a thinner shell, where the first absorption of stellar photons takes place. This enables an accretion flow to break through this region of direct stellar irradiation feedback more easily. Secondly, the feedback by radiation from dust grains, which actually stops the accretion in the spherically symmetric case, will be strongly reduced, because the majority of the radiative flux 

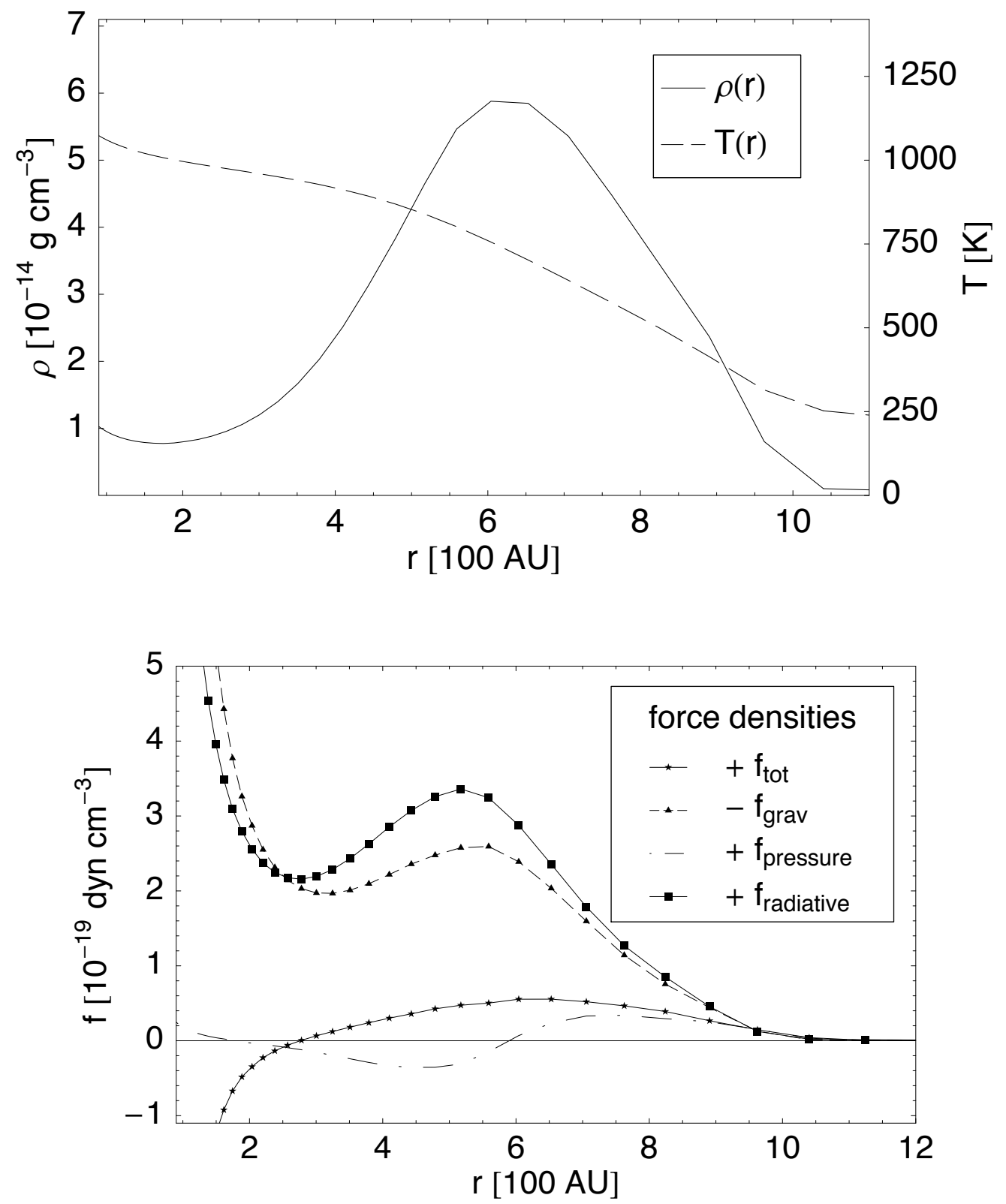

Fig. 8.- : Snapshot of radial force densities (lower panel) and the density and temperature profile (upper panel) in the innermost core region taken from the collapse simulation of a $M_{\text {core }}=120 \mathrm{M}_{\odot}$ pre-stellar core at $20 \mathrm{kyr}$ corresponding to a proto-stellar mass of about $M_{*}=25 \mathrm{M}_{\odot}$. Due to the superior radiative force the spherically symmetric accretion models yield an upper mass limit of the final star of $M_{*}^{1 \mathrm{D}}<40 \mathrm{M}_{\odot}$. 
from the irradiated inner rim of the disk will escape in the vertical direction through the optically thin disk atmosphere and therefore does not interact with the radially inward-streaming accretion flow. The different kinds of radiative feedback in spherical symmetry as well as in an axially symmetric disk geometry are illustrated in the final discussion Sect. 6.

Analogously to the discussion of the spherical symmetric simulations, we present in the following the results of axially and midplane symmetric simulations of the collapse of rotating massive pre-stellar cores. Before being able to scan the parameter space of different initial core masses (Sect. 5.2), we determine the required resolution in convergence runs (Sect. A.2) and fix the radius $r_{\text {min }}$ of the central sink cell in various simulations (Sect. 5.1).

\subsection{Parameter scan of the size of the sink cell: The influence of the dust sublimation front}

\subsubsection{Simulations}

In the following, we study the influence of the radius $r_{\min }$ of the inner sink cell, which equals the inner computational boundary, on the resulting accretion rate onto the evolving massive star. We check this dependency in four simulations with a radius of the inner sink cell of $r_{\min }=1,5,10$, and $80 \mathrm{AU}$. The initial conditions and numerical parameters of these runs are described in Sect. 3 and the simulations are performed for an initial core mass of $M_{\text {core }}=60 \mathrm{M}_{\odot}$. We follow the long-term evolution of the runs for at least $92 \mathrm{kyr}$. The resulting accretion rate onto the forming star as well as the mass growth of the central star are displayed in Fig. 9.

\subsubsection{Conclusions}

In the spherically symmetric models, we conclude that the numerical results do not depend on the radius $r_{\text {min }}$ of the central sink cell unless it is smaller than the dust sublimation radius $r_{\text {subl }}$ from the point in time at which the radiative force overcomes gravity. These results cannot easily be transferred to the axially symmetric disk configuration. Including centrifugal forces, which compensate the gravity in the disk region, the chosen location $r_{\min }$ of the inner boundary of the computational domain influences the resulting accretion rate in two distinguishable effects:

Due to the fact that the circumstellar disk is growing in time from the inside outwards, a smaller sink cell leads to an earlier onset of the disk formation phase during the simulation. In other words, a fluid package with an initial position at $\left(r_{i}, \theta_{i}\right)$ and an initial rotation of $\Omega_{i}$ hold

a centrifugal radius of $r_{\text {cent }}=\frac{\Omega_{i}^{2} r_{i}^{4}}{G M\left(r_{i}\right)} \sin ^{2}\left(\theta_{i}\right)$ with the included mass $M\left(r_{i}\right)$, see Eq. (12). If this centrifugal radius is smaller than the sink cell radius $r_{\min }$, the fluid package is accreted onto the forming star during the so-called free fall epoch at the beginning of the simulation. This effect is associated with the gas physics (hydrodynamics) of the pre-stellar core, because the gas represents 

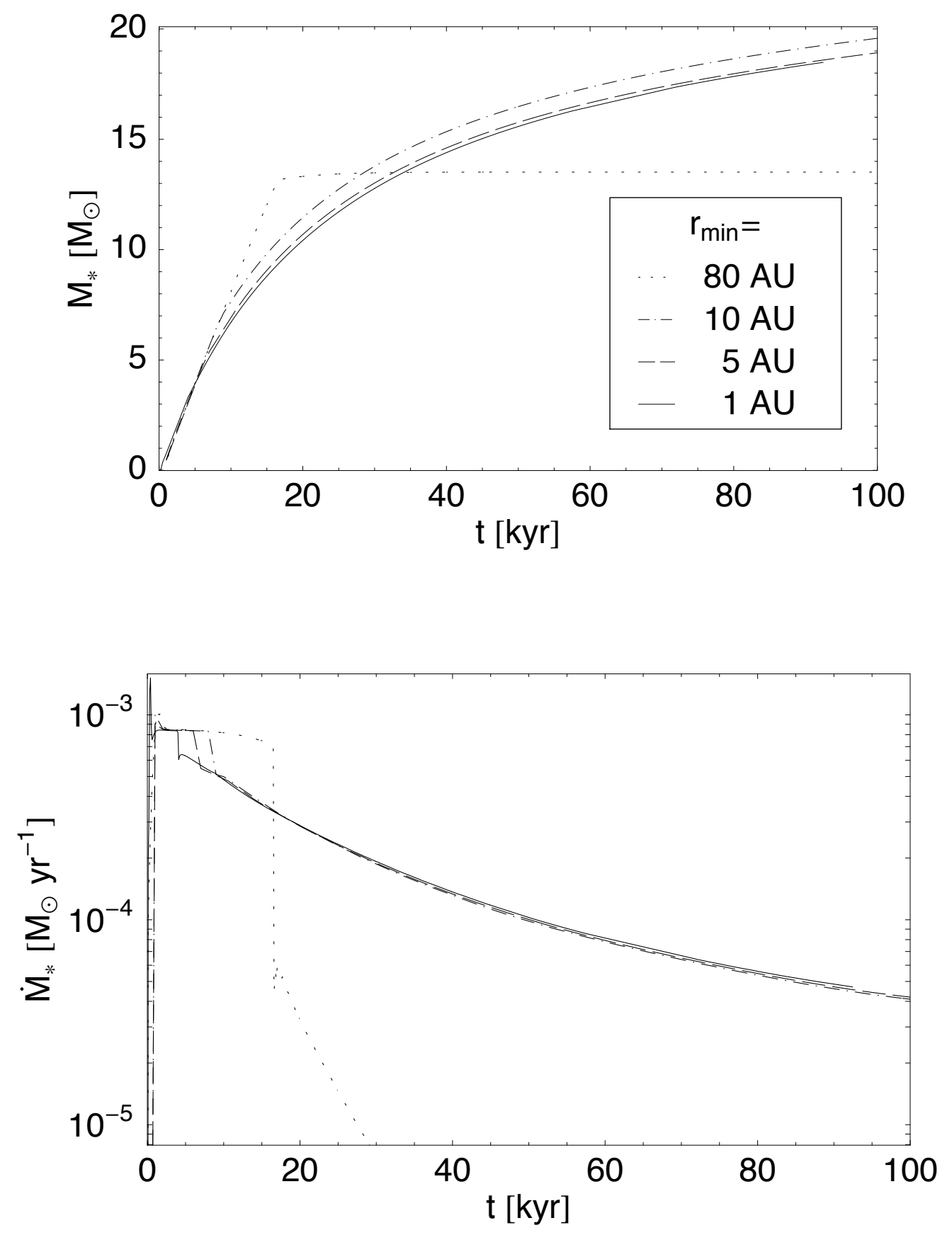

Fig. 9.- : Stellar mass $M_{*}$ (upper panel) and accretion rate $\dot{M}_{*}$ (lower panel) as a function of time $t$ for different radii $r_{\min }$ of the central sink cell in the collapse simulation of a rotating $60 \mathrm{M}_{\odot}$ pre-stellar core. 
$99 \%$ of the mass of the pre-stellar core. A second important effect depending on the chosen sink cell radius is related to the dust and therefore to the radiation physics. The region in the vicinity of the forming massive star will be heated up to temperatures beyond the dust sublimation temperature. Therefore a gap is formed between the central star and the dust disk. Under the assumption that the absorption by gas in this gap is much smaller than the absorption by dust grains behind the dust sublimation front the inner rim of the dust disk determines the region of the first stellar radiative impact onto the accretion flow. Also the most important radiative feedback due to thermal re-emission by dust grains sets in directly behind this irradiated heated region. Contrary to the spherically symmetric case, the high-density disk region bypasses most of the re-emitted radiation into the vertical direction, i.e. the total radiation field is composed of the isotropic stellar irradiation and a highly anisotropic (secondary) thermal radiation field.

Fig. 9 illustrates clearly both, the mass and the radiative effect, related to an artificial inner cut-off of the gas and the dust disk respectively: As expected, the duration of the so-called free fall phase shortens with the radius $r_{\min }$ of the sink cell. This behaviour can fortunately be estimated analytically given the sink cell radius and the initial conditions of the pre-stellar core to account for the overestimation of the final mass of the forming star, if necessary. Moreover, this effect of the artificial inner rim of the gas disk results on the one hand in an overestimation of the final mass of the central star by approximately $1 \mathrm{M}_{\odot}$ or below (upper panel of Fig. 9), but on the other hand influences the proceeding radiation hydrodynamic interactions in its environment only marginally (lower panel of Fig. 9). The corresponding accretion rates after the disk formation are not influenced at all. This result is quite reasonable keeping in mind that the balance of radiative and gravitational forces can be described in first order by the luminosity to mass ratio $L_{\text {tot }} / M_{*}$ of the central massive star, which only changes marginally with another choice of the size of the central sink cell.

But the radiative impact due to the artificial cut-off of the inner dust disk regime for sink cell radii larger than the dust sublimation front has dramatic effects. In the case of $r_{\text {min }}=80 \mathrm{AU}$ the artificial shift of the region of the dust radiation interactions terminates the short disk accretion phase abruptly, leading to a completely wrong evolution of the central star, the disk as well as the large scale environment. The reason for this dramatic change in the radiation physics is that the lower density region of the circumstellar disk at $80 \mathrm{AU}$ is (in contrast to the real inner rim of the dust disk at roughly $20 \mathrm{AU}$ ) not opaque enough to generate a strong anisotropy of the thermal radiation field. Therefore the strong isotropic part of the thermal radiation field is able to stop the accretion analogous to the spherically symmetric flow calculations.

Due to the importance of this inner core region for the associated interaction of the radiation with the accretion flow it seems to be unavoidable to include the whole dust disk down to its inner rim in the computational domain (cp. Fig. 10). This defines an upper limit of the radius $r_{\min }$ of the central sink cell, which has to be smaller than the dust sublimation radius $r_{\text {subl }}$ in the midplane from that point in time at which the radiative force has grown to a competetive magnitude compared to the viscous force driving the accretion flow. Subsequent simulations meet 
this concern by using an adequate central sink cell radius of $r_{\min }=10 \mathrm{AU}$. Otherwise, for an inner sink cell radius $r_{\text {min }}$ larger than the dust sublimation radius $r_{\text {subl }}$, the region of radiative feedback is artificially shifted to higher radii including a strong decrease in density, opacity, and gravity. The resulting strong heating of the disk region behind the radius $r_{\min }>r_{\text {subl }}$, which 'realistically' would be shielded from the stellar irradiation by the inner parts of the disk, leads to a diminishment of the shielding/shadowing property of the massive accretion disk. In case the radiation field retained therefore its isotropic character in major parts, the radiation pressure stops the emerging disk accretion phase, similar to the spherically symmetric case.

This dependency of the radiation pressure on the radius of the sink cell explains also the abrupt end of the accretion phase in the simulations by Yorke \& Sonnhalter (2002). They presented simulations of collapsing pre-stellar cores of $M_{\text {core }}=30 \mathrm{M}_{\odot}, 60 \mathrm{M}_{\odot}$ and $120 \mathrm{M}_{\odot}$. The radius of their inner sink cell was chosen proportional to the initial mass of the core to be 40, 80, and $160 \mathrm{AU}$ respectively. As shown in this parameter scan (cp. Fig. 9) such huge sink cells lead to an artificial cut-off of the dust disk and result therefore in unphysically strong radiative feedback. Therefore, we are definitely sure that this yields also the abrupt and early end of the accretion phase in the simulations by Yorke \& Sonnhalter (2002).

Contrary to our conclusion, Krumholz et al. (2009) stated that the much longer accretion phases in their own simulations compared to Yorke \& Sonnhalter (2002) are a result of non-axially symmetric modes in the outflow region. The physical argument in that case is that their simulations show a so-called "3D radiative Rayleigh-Taylor instability" in the radiation pressure driven outflow, which results in further mass accretion onto the circumstellar disk from the bipolar direction instead of a steady outflow feature. In axially symmetric simulations the feeding of the disk would therefore not be possible. Our axially symmetric collapse simulations, presented in the following section, show a stable radiation pressure driven outflow and the forming circumstellar disk gains enough mass from the huge mass reservoir of the envelope to maintain its shielding property over several free fall times, in fact over a longer period ever simulated in previous research studies.

\subsection{Parameter scan of the initial pre-stellar core mass: Breaking through the upper mass limit of spherically symmetric accretion}

\subsubsection{Simulations}

The spherically symmetric (one-dimensional) collapse simulations of massive pre-stellar cores yield a maximum stellar mass of less than $40 \mathrm{M}_{\odot}$ independent of the initial core mass $M_{\text {core }} \geq 60 \mathrm{M}_{\odot}$ due to radiative feedback. We attack this radiation pressure barrier in two-dimensional axially and midplane symmetric circumstellar disk geometry now. The implications of this change of geometries are illustrated at full length in the final discussion Sect. 6. We performed four simulations with the default initial conditions described in Sect. 3 and the fixed numerical parameters presented in Sect. A.2 and 5.1, namely a maximum resolution of $1.27 \mathrm{AU}$ x $1.04 \mathrm{AU}$, and an inner sink cell radius 


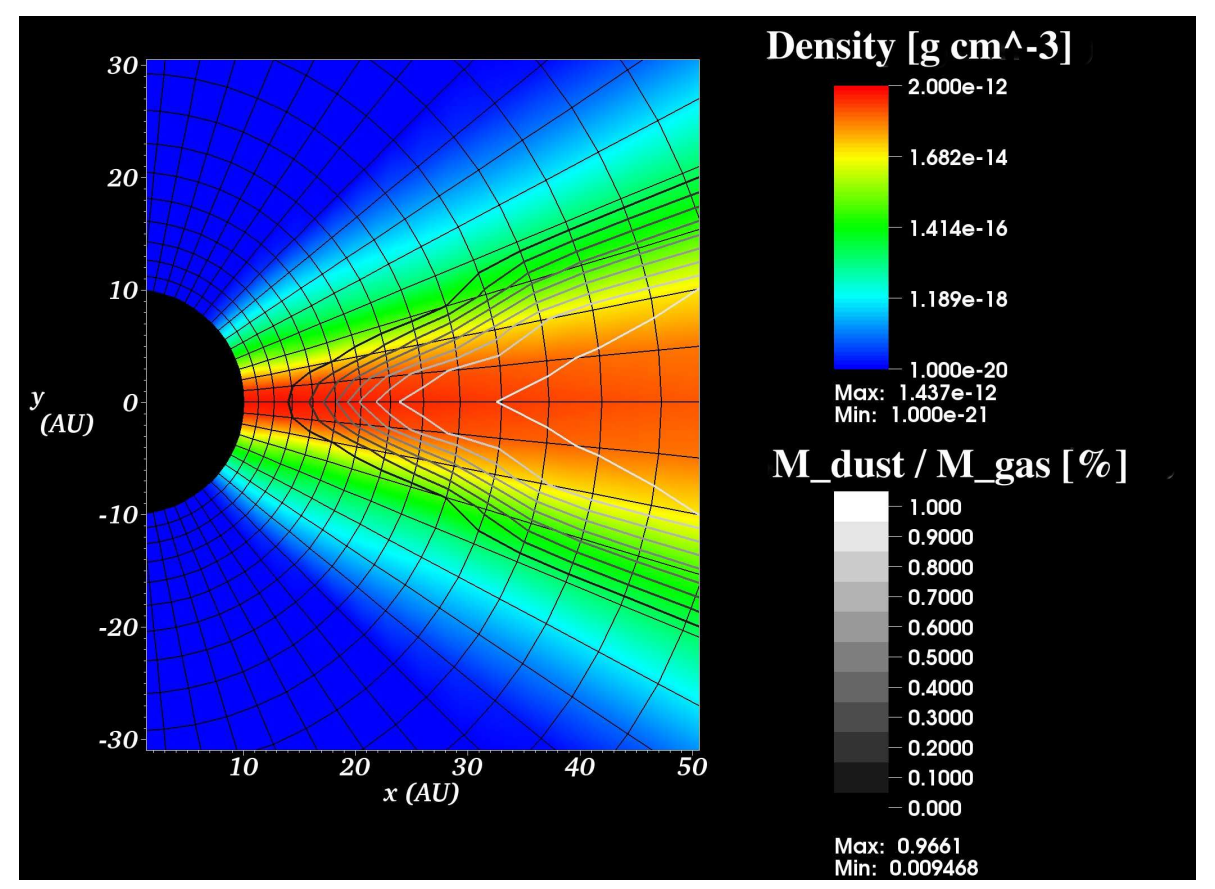

Fig. 10.- : Resolving the dust sublimation front. The image shows a snapshot at $100 \mathrm{kyr}$ after start of the collapse of a $120 \mathrm{M}_{\odot}$ pre-stellar core.

Color: Gas density from $10^{-20}$ up to $2 * 10^{-12} \mathrm{~g} \mathrm{~cm}^{-3}$ in logarithmic scale.

Contour-lines: Dust to gas mass ratio from 0 up to $1 \%$ in linear scale.

The zoom-in illustrates the fact that the dust sublimation front is resolved smoothly over several (here roughly 8) grid cells in the radial direction. 
of $10 \mathrm{AU}$. The different initial core masses of $M_{\text {core }}=60 \mathrm{M}_{\odot}, 120 \mathrm{M}_{\odot}, 240 \mathrm{M}_{\odot}$, and $480 \mathrm{M}_{\odot}$ are chosen analog to the scan of the initial core mass parameter in the spherically symmetric case. The resulting accretion histories as a function of the actual stellar mass are displayed in Fig. 11.

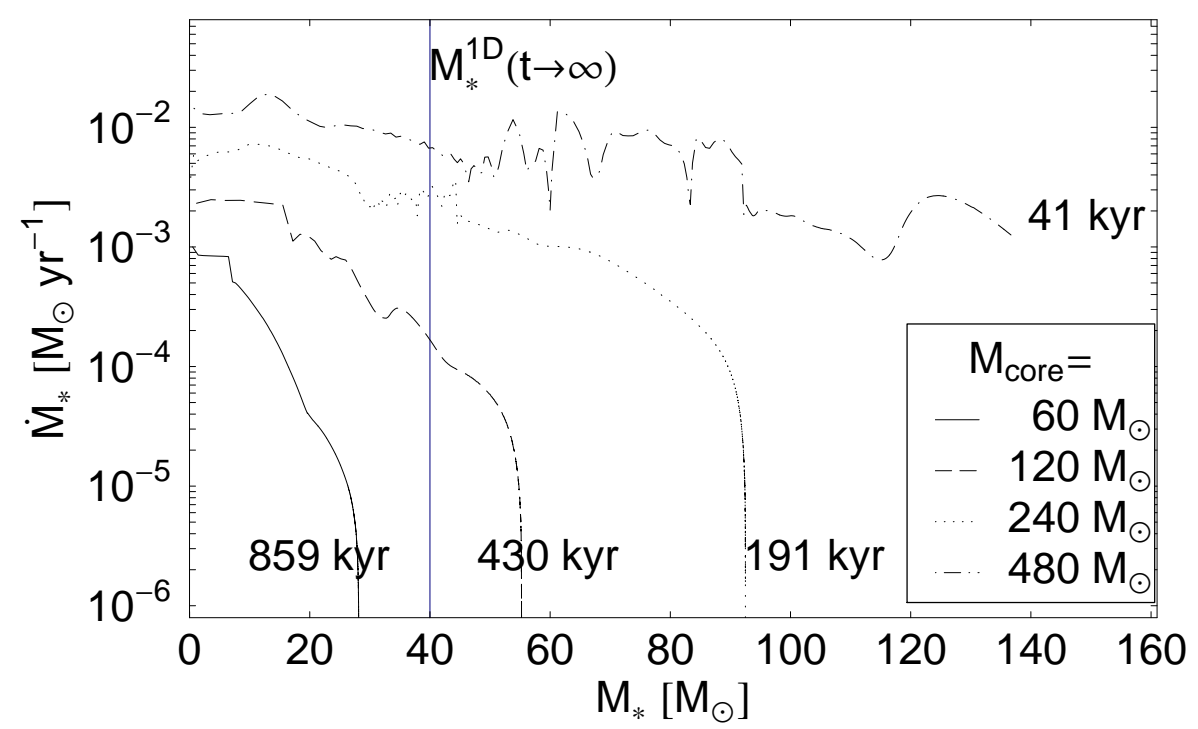

Fig. 11.- : Accretion rate $\dot{M}_{*}$ as a function of the actual stellar mass $M_{*}$ for four different initial core masses $M_{\text {core }}=60 \mathrm{M}_{\odot}, 120 \mathrm{M}_{\odot}, 240 \mathrm{M}_{\odot}$, and $480 \mathrm{M}_{\odot}$. Also the periods of accretion are mentioned for each run. The vertical line marks the upper mass limit found in the spherically symmetric accretion models. The collapse models of slowly rotating pre-stellar cores clearly break through this upper mass limit of the final star of $M_{*}^{1 \mathrm{D}}<40 \mathrm{M}_{\odot}$.

\subsubsection{Conclusions}

As expected, the lowest mass case of $M_{\text {core }}=60 \mathrm{M}_{\odot}$ results finally in a less massive central star than the corresponding run in spherical symmetry (without rotation) simply due to the fact that the additional angular momentum results in centrifugal forces, which counteracts the accretion flow driven by gravity and viscosity. In face of this additional centrifugal forces, for higher mass pre-stellar cores the slowed down accretion flux breaks easily through the upper mass limit of the final star of $M_{*}^{1 \mathrm{D}}<40 \mathrm{M}_{\odot}$ found in the spherically symmetric accretion models!

The reason for that breakthrough can be displayed by a closer look at the driving force densities in the evolved pre-stellar core, plotted in Figs. 12 to 17. All figures represent a snapshot of the $M_{\text {core }}=120 \mathrm{M}_{\odot}$ case at $60 \mathrm{kyr}$ after start of the simulation. At this point in time, the actual mass of the central massive star is roughly $40 \mathrm{M}_{\odot}$, representing the spherically symmetric upper mass 
limit found in previous simulations (Sect. 4.2). In contrast to the spherically symmetric models, the geometry of the proto-stellar environment can now be divided into a very dense circumstellar disk and the lower density envelope. We visualized exemplary the actual density, velocity, and the acting forces in the radial direction for both regimes, Figs. 12 to 14 for the midplane of the accretion disk, Figs. 15 to 17 for a polar angle of $30^{\circ}$ above the midplane. In the midplane the gravity and centrifugal force are one to two orders of magnitude higher than the thermal pressure and up to three orders of magnitude higher than the radiative and viscous force. The upper panel of Fig. 12 shows three individual regions of the midplane layer, in between the sign of the total force density changes. The gravity dominates the individual forces for the outer core regions (above 3000 AU) leading to a steady accretion flow onto the inner core region (Figs. 12 and 13). In the very inner part of the core around the massive star (below 200 AU) the gravity is balanced by the centrifugal force and in small part by the thermal pressure (Fig. 13). In this region, which we will refer to as the disk region hereafter, the shear viscosity yields a quasi-stationary accretion flow through the disk, which clearly exceeds the radiative force (Fig. 14). In between this disk region $(<200 \mathrm{AU})$ and the global in-fall region ( $>3000$ AU) the mass flux describes transient oscillations, because gravity, centrifugal forces and thermal pressure are not in equilibrium yet, as it is the case for the mass finally arriving the disk region. Although the total force density, displayed in the upper panel of Fig. 12 is directed in the outward direction between 200 and $3000 \mathrm{AU}$, the mass in this region is still in an inward motion (cp. Fig. 12, lower panel), i.e. the mass flow through the pre-stellar core is feeding the circumstellar accretion disk. The viscous force in the accretion disk is able to drive a steady accretion flow towards the evolving massive star of $40 \mathrm{M}_{\odot}$, because the radiative force is one to two orders of magnitude lower in this dense disk region than in the low density envelope (cp. Figs. 14 and 17). Observations of such a large scale flattened structure with a potentially embedded small scale accretion disk are i.e. described in Fallscheer et al. (2009) and Beuther et al. (2009).

At an polar angle of $30^{\circ}$ above the midplane this strong radiative force already accelerates the remnant mass in the radially outward direction through mostly the entire pre-stellar core (Fig. 16). Only at the outer rim of the core the previous in-fall motion is still visible. This distribution of the individual force densities confirms in high detail the expected result of the anisotropy of the thermal radiation field. Most of the radiative flux from the irradiated inner rim of the disk is bypassed in the vertical direction through the optically thin atmosphere of the circumstellar disk. Meanwhile, the accretion flow is reduced compared to the one-dimensional gravitational in-fall to a steady stream driven by the viscous properties of the accretion disk. In the envelope region of the pre-stellar core the radiative force reverts the in-fall motion and depletes the stellar surrounding similar to the spherically symmetric accretion models (cp. the corresponding individual force distributions in Figs. 15 and 8).

In these axially and midplane symmetric disk accretion models no upper mass limit of the final star is detected so far, but the star formation efficiency declines for higher mass cores. The proceeding depletion of the envelope by radiative forces finally leads to a decrease of the density 
in the midplane and the disk looses its shielding property. Without this shielding the radiation pressure starts to accelerate the remnant material in the outward direction.

\section{Discussion}

\subsection{Radiation pressure feedback in a nutshell}

Since the isotropic and anisotropic feature of the spherical and disk accretion scenario at the dust sublimation front is of such a great importance in our simulation results, we illustrate these key attributes in more detail: In this research study, we investigated the influence of the stellar environment onto the radiation pressure problem in the formation of massive stars. We studied the accretion flow onto a high-mass star in a monolithic pre-stellar core collapse picture, as recommended by Whitney (2005) and McKee \& Ostriker (2007). Under this assumption, the theoretical description of the accretion process onto a massive star has to deal with the interaction between the exerted radiation by the forming star with the accretion flow of gas and dust (Shu et al. 1987). In a perfectly spherically symmetric collapse, this interaction potentially stops the accretion onto the star entirely. In the static limit, radiation pressure overcomes gravity at the so-called generalized Eddington barrier

$$
\frac{L_{*}}{M_{*}}=\frac{4 \pi G c}{\kappa_{*}},
$$

where $L_{*}, M_{*}$ and $\kappa_{*}$ denote the stellar luminosity, the stellar mass, and the dust opacity respectively, $G$ is the gravitational constant and $c$ is the speed of light. But the collapse of a pre-stellar core is far from being a static problem. The momentum transfer from the absorbed photons first has to slow down the in-falling envelope. For simplification purposes, we can divide the radiation pressure feedback into two components, as illustrated in Fig. 18. The first exchange of momentum takes place when the irradiation from the massive star is absorbed by the dust grains of the surrounding, i.e. behind the dust sublimation radius. The strongest force will thereby be produced by photons with shorter wavelengths, because they have a higher absorption probability and are more energetic. We will call this first interaction "UV feedback" abbreviated, although the frequency dependence of the broad stellar black body spectrum is clearly not negligible. Afterwards, these heated regions emit most of the photons at the dust temperature, yielding a much longer wavelength and a much longer mean free path than the direct stellar light. The interaction of this radiation with the enclosed gas and dust is therefore referred to as "IR feedback". Our spherically symmetric collapse simulations confirm the outcome of previous studies that it is essentially the IR feedback that stops the accretion flow onto the forming star in spherical symmetry. Although each IR photon transfers less momentum to the dust than the highly energetic stellar UV photons, the thermal dust emission acts onto the accretion flow on a much larger volume than the spatially confined absorption region of the stellar irradiation. Additionally the optical depth of the envelope decreases towards longer wavelength, so the IR feedback conteracts the accretion flow

in the outer core regions yielding less gravity. Different approaches to overcome this barrier for 

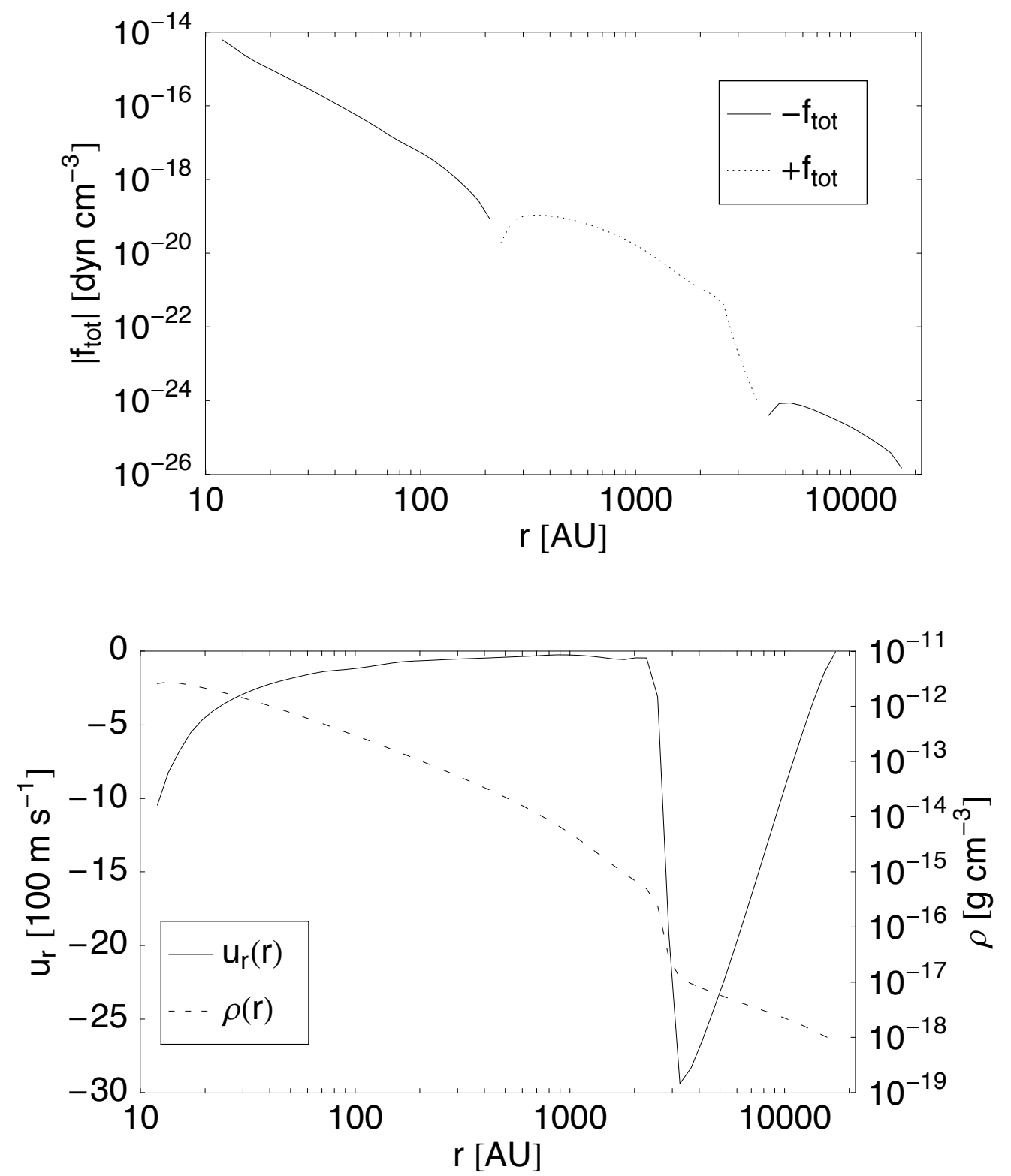

Fig. 12.- : Total force density $\left|f_{\text {tot }}(r)\right|$ (upper panel) as well as density $\rho(r)$ and radial velocity $u_{r}(r)$ (lower panel) as a function of radius $r$ through the disk's midplane. The snapshot was taken at $60 \mathrm{kyr}$ after start of the simulation, corresponding to a central stellar mass of roughly $40 \mathrm{M}_{\odot}$. The individual force densities along this line of sight through the total and the inner core region are displayed in Figs. 13 and 14. 


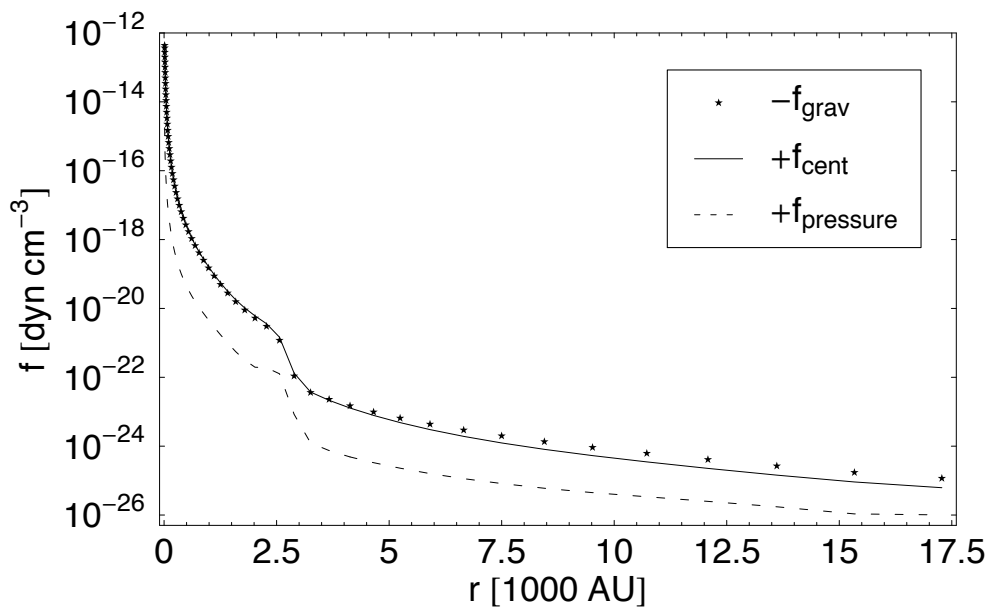

Fig. 13.- : Gravity, centrifugal, and thermal pressure force as a function of radius through the disk's midplane. The snapshot was taken at 60 kyr after start of the simulation, corresponding to a central stellar mass of roughly $40 \mathrm{M}_{\odot}$. The radiative and viscous forces are orders of magnitude smaller than the illustrated ones, but become important in the inner disk region, where the stronger forces are in equilibrium. The radiative and viscous force densities along this line of sight through the inner core region are displayed in Fig. 14.

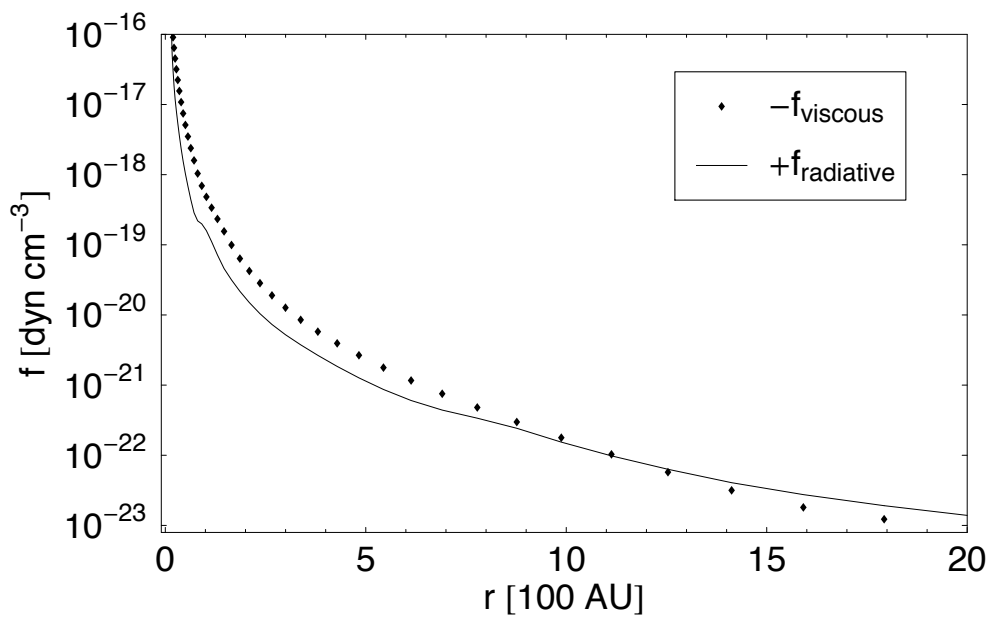

Fig. 14.- : Viscous and radiative force density of the inner core region as a function of radius through the disk's midplane. The snapshot was taken at $60 \mathrm{kyr}$ after start of the simulation, corresponding to a central stellar mass of roughly $40 \mathrm{M}_{\odot}$. 

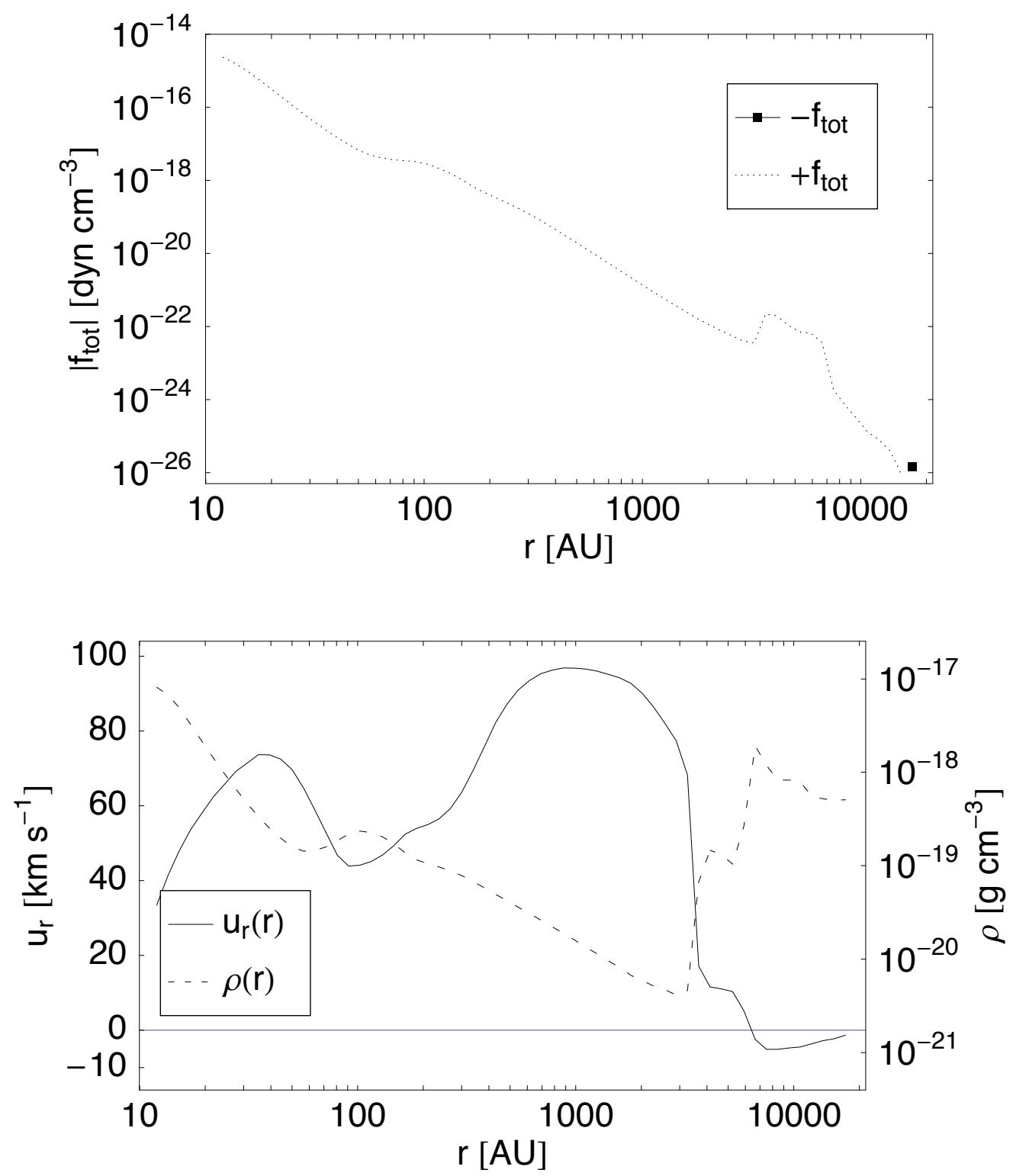

Fig. 15.- : Total force density $\left|f_{\text {tot }}(r)\right|$ (upper panel) as well as density $\rho(r)$ and radial velocity $u_{r}(r)$ (lower panel) as a function of radius $r$ at $30^{\circ}$ above the disk's midplane. The snapshot was taken at $60 \mathrm{kyr}$ after start of the simulation, corresponding to a central stellar mass of roughly $40 \mathrm{M}_{\odot}$. The individual force densities along this line of sight through the total and the inner core region are displayed in Figs. 16 and 17. 


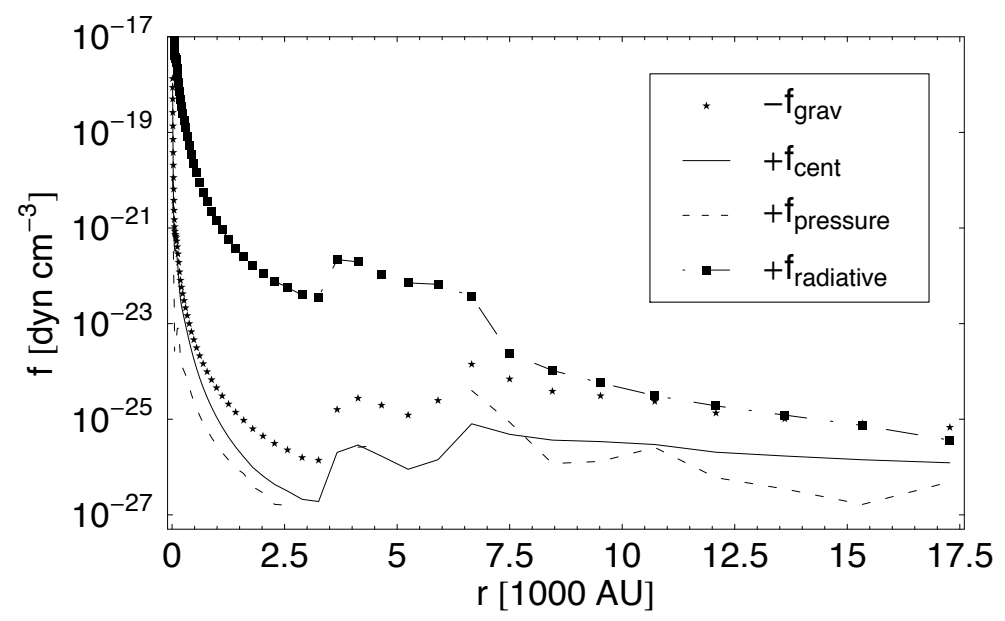

Fig. 16.- : Gravity, centrifugal, thermal pressure, and radiative forces as a function of radius at $30^{\circ}$ above the disk's midplane. The snapshot was taken at $60 \mathrm{kyr}$ after start of the simulation, corresponding to a central stellar mass of roughly $40 \mathrm{M}_{\odot}$. The individual force densities along this line of sight through the inner core region are displayed in Fig. 17.

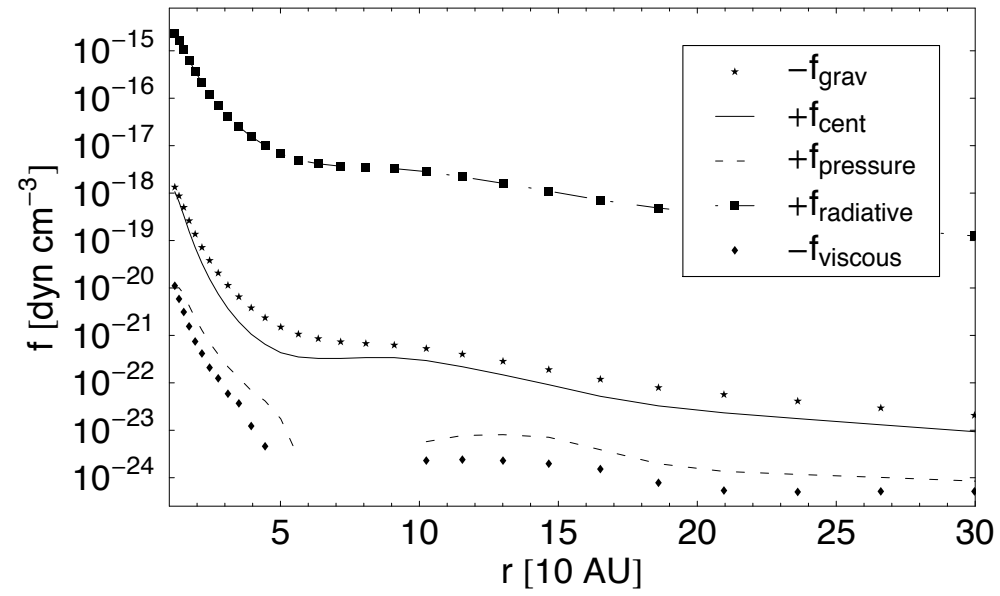

Fig. 17.- : Gravity, centrifugal, thermal pressure, radiative, and viscous force density of the inner core region as a function of radius at $30^{\circ}$ above the disk's midplane. The snapshot was taken at $60 \mathrm{kyr}$ after start of the simulation, corresponding to a central stellar mass of roughly $40 \mathrm{M}_{\odot}$. 


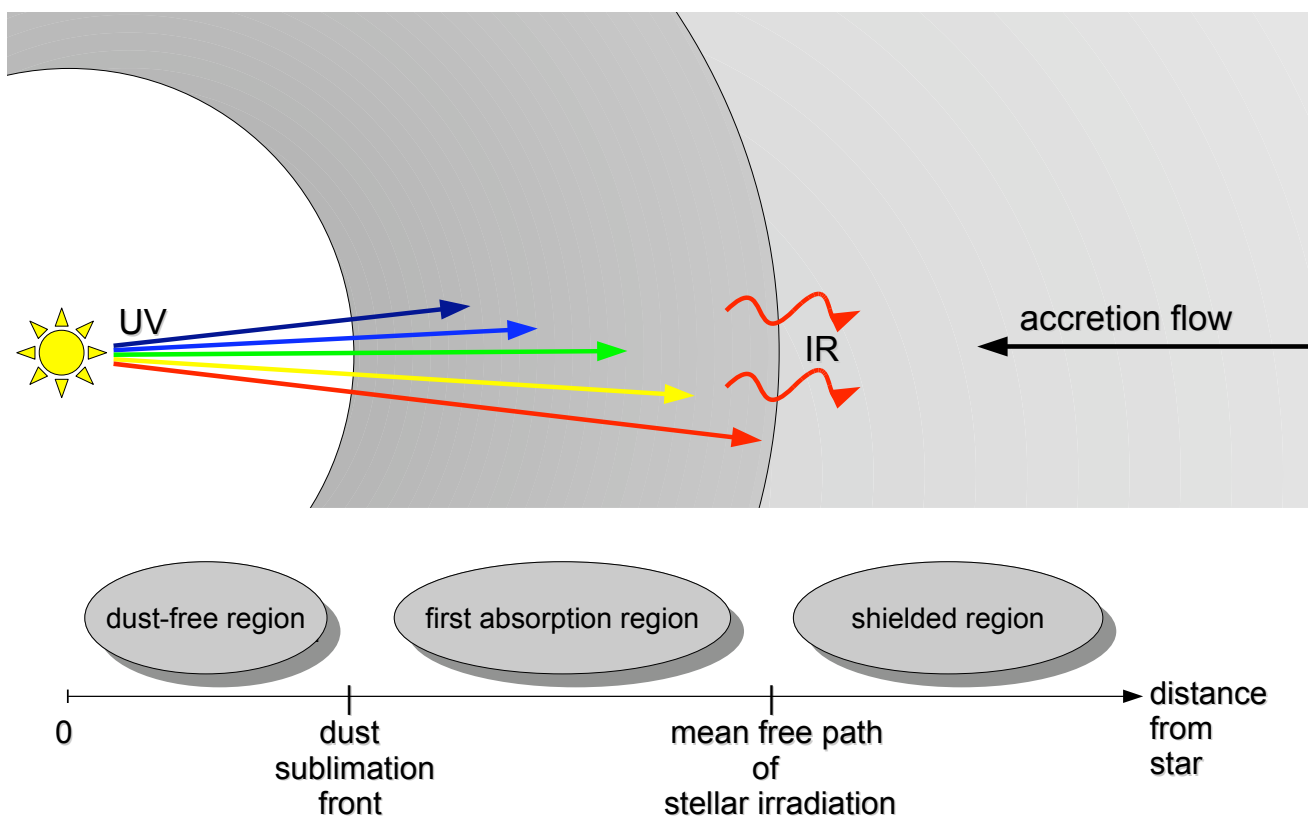

Fig. 18.- : Schematic view of the radiative forces onto the accretion flow in spherical symmetry. The radiative feedback is divided into direct stellar irradiation and secondary re-emitted photons by dust grains.

spherically symmetric accretion flows onto massive stars were considered in the past. The generalized Eddington barrier depends only on the stellar evolution $\left(L_{*} / M_{*}\right)$ and on the dust properties $\left(\kappa_{*}\right)$. Wolfire \& Cassinelli (1987) studied the necessary change of dust properties to enable further accretion, but the restrictions they derived seem to be unrealistic.

Without a doubt, star formation is rarely a perfectly spherically symmetric problem. Initial angular momentum of the collapsing pre-stellar core leads to the formation of a circumstellar disk as well as polar cavities. Compared to the case of spherically symmetric accretion, the disk geometry changes the radiation pressure feedback dramatically, see Fig. 19. Going from a spherically symmetric in-fall to an axially symmetric disk geometry can help to overcome both - the UV as well as the IR - radiation pressure feedback: Developing radiation hydrodynamical (Klahr \& Bodenheimer 2003), magneto-rotational (Balbus \& Hawley 1991; Hawley \& Balbus 1991; Balbus 2003) and selfgravitating instabilities (Yang et al. 1991; Laughlin \& Bodenheimer 1994; Bodenheimer 1995) in the accretion disk will transfer angular momentum to outer radii allowing a steady mass accretion radially inward. The additional ram pressure in the radiatively shielded parts of the disk will possibly push the mass over the thin shell of the UV feedback (Nakano 1989). Secondly, and most important, the irradiated and therefore heated regions of the disk will mainly cool in the vertical direction through the optically thin disk's atmosphere, strongly restraining the IR radiation pressure in the radial direction. If the latter process occurs at the innermost part of the disk so that the radiation can escape directly through the bipolar cavity, this effect is also known as the so-called 


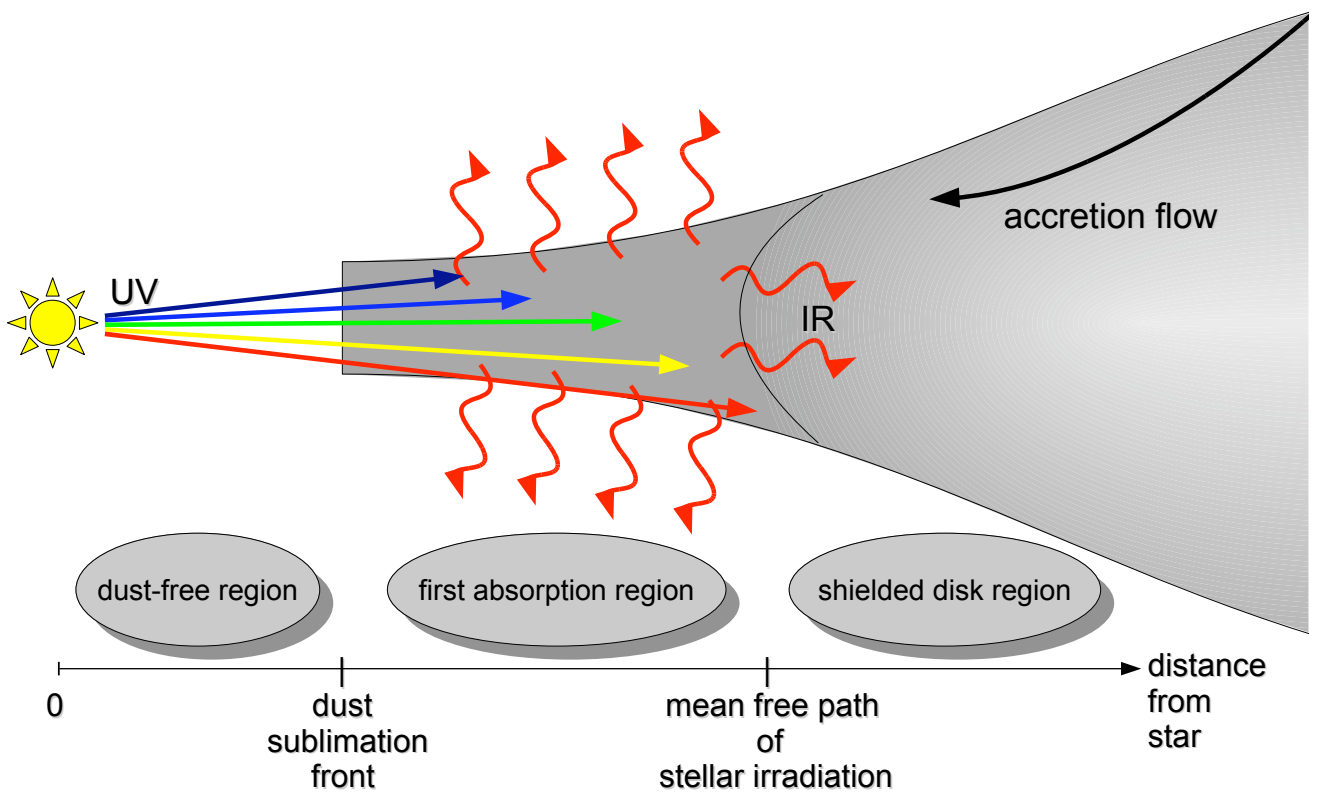

Fig. 19.-: Schematic view of the "UV"- and "IR"-component of the radiation pressure acting in an axially symmetric circumstellar disk geometry.

\section{'flashlight-effect' (Yorke \& Sonnhalter 2002; Krumholz et al. 2005).}

The interaction of the radiation with the accretion flow is very sensitive to the numerical treatment of radiation transport. The FLD approximation, which is a standard technique in modern radiation hydrodynamics codes for astrophysical fluid flows, fails to compute the correct flux between the first transition region from the dust depleted zone around the massive star and the optically thick disk leading to an incorrect temperature distribution in the irradiated regions (see e.g. Yorke \& Krügel 1977; Boley et al. 2007). Also simplifying the stellar black body spectrum by using frequency averaged Planck mean opacities leads to a thinner shell of the direct stellar irradiation feedback and a stronger heating of the corresponding dust, which afterwards yields a higher IR feedback. Hence, accounting for the frequency dependence of the stellar spectrum seems to be a crucial point.

The most violent interaction of the stellar irradiation with the accretion flow takes place at and directly behind the first absorption peak. The location of the first absorption layer is represented by the dust sublimation front, where the local dust temperature falls below the evaporation temperature of the dust grains. A systematic study of the radiation pressure feedback on the formation of massive stars therefore implies the need to resolve the ongoing radiation and accretion physics down to the dust sublimation front. A formation of massive stars by breaking through the ionization boundary into regions of sublimated dust grains was studied for spherically symmetric accretion flows (Keto 2003) as well as for two-dimensional effects in the so-called small radius limit (Jijina \& Adams 1996). Aside from the important contribution of the proceeding physics at the 
dust sublimation front, no previous non-spherically symmetric numerical research has been done so far, presumably due to resolution issues.

\subsection{Comparison to previous numerical research in the field}

In our simulations the star in the center of the accretion disk grows far beyond the upper mass limit found in the case of spherical accretion. Indeed, the final massive stars are the most massive stars ever formed in a multi-dimensional radiation hydrodynamics simulation so far. The quantitative final results as well as a comparison to previous multi-dimensional radiation hydrodynamics studies is presented in Table 5. The research studies clearly differ in the evolutionary time simulated. We improved this by roughly an order of magnitude. The $20 \mathrm{kyr}$ and $75 \mathrm{kyr}$ of evolution in Krumholz et al. (2007) and Krumholz et al. (2009) represent approximately one-third and slightly more than one free fall time of the pre-stellar core respectively. Despite the frequency dependent radiation transport and the high resolution down to 1.27 AU in our simulations, we are able to follow the evolution of the accreting system up to 14, 10, 7, and 2 free fall times for an initial core mass of $60,120,240$, and $480 \mathrm{M}_{\odot}$ respectively, including the whole stellar accretion phase. To state this clearly, this is only possible due to our self-restriction to axial symmetry in these runs. In the simulations by M. Krumholz further accretion seems to be the natural continuation of the runs. Our simulation series of the disk accretion scenario with varying initial core masses shows a decrease of the star formation efficiency towards higher mass cores as a result of the growing radiation pressure feedback.

Simultaneously to the bypass of the thermal radiation by the massive accretion disk, a stable wide-angle bipolar outflow with velocities of the order of $100 \mathrm{~km} \mathrm{~s}^{-1}$ is launched by the radiation pressure. In these axially symmetric simulations, we did not detect any evidence for a radiative instability of these outflow regions, like observed in the simulations by Krumholz et al. (2009). Following the explanatory notes in Krumholz et al. (2009), this is due to the fact, that this instability requires non-axial symmetric modes to occur. A detailed study of this regime seems to be necessary to clearly understand the underlying physics of this requirement. On the other hand, our simulations show a strong release of radiation pressure in the bipolar direction, growing in angle with time, which fits to the observed broadening of outflows in massive star forming regions (Beuther \& Shepherd 2005). But to state this clearly, we are sure that a complete description of the jet or outflow physics cannot be done without taking care of the dominant magnetic field effects.

Fig. 20 shows the time dependent fractions of masses, divided into the mass inside the computational domain, the mass of the forming star (from the flux over the inner boundary), and the mass loss by the radiation pressure driven outflow (from the flux over the outer boundary).

The different epochs of the collapse of the $120 \mathrm{M}_{\odot}$ pre-stellar core are illustrated in Fig. 21. The subfigures display the initial condition, the disk formation and evolution, the outflow launching, and the end of the accretion phase in several snapshots of the density structure. The corresponding 
Table 5. Overview of multi-dimensional radiation hydrodynamics simulations of massive star formation

\begin{tabular}{|c|c|c|c|c|}
\hline Authors & $\begin{array}{c}M_{\text {core }} \\
\left(\mathrm{M}_{\odot}\right)\end{array}$ & $\begin{array}{c}t_{\text {end }} \\
(\mathrm{kyr})\end{array}$ & $\begin{array}{c}M_{*} \\
\left(\mathrm{M}_{\odot}\right)\end{array}$ & $\begin{array}{l}\text { SFE } \\
(\%)\end{array}$ \\
\hline \multirow{3}{*}{ Yorke \& Sonnhalter (2002) } & 30 & 25 & 31.6 & $\cdots$ \\
\hline & 60 & 45 & 33.6 & $\cdots$ \\
\hline & 120 & 70 & 42.9 & $\cdots$ \\
\hline \multirow{3}{*}{ Krumholz et al. (2007) } & $100(\mathrm{~A})$ & $20^{+}$ & $5.4(+3.4)$ & $>5.4$ \\
\hline & $100(\mathrm{~B})$ & $20^{+}$ & $8.9(+2.4)$ & $>8.9$ \\
\hline & 200 & $20^{+}$ & $8.6(+6)$ & $>8.6$ \\
\hline Krumholz et al. (2009) & 100 & $75^{+}$ & $41.5+29.2(+28.3)$ & $>70.7$ \\
\hline \multirow{4}{*}{ This study } & 60 & 939 & 28.2 & 47.0 \\
\hline & 120 & 489 & 56.5 & 47.1 \\
\hline & 240 & 226 & 92.6 & 38.5 \\
\hline & 480 & $41^{+}$ & $137.2(+67.8)$ & $>28.5$ but $<42.7$ \\
\hline
\end{tabular}

Note. - The columns from left to right state the authors, the initial pre-stellar core mass, the evolutionary time simulated, the final star mass, as well as the corresponding star formation efficiency. A "+" in the $t_{\text {end }}$ column means that the accretion phase is not simulated until the end yet. In the case of the simulation by M. Krumholz, only the formation of the most massive stars are considered here; all other stars formed have masses below $1 \mathrm{M}_{\odot}$. In the case of Krumholz et al. (2007) the "(A)" and "(B)" in the $M_{\text {core }}$ column mark the usage of different perturbation fields of the initial state (same labels as in the original paper) and the $M_{*}$ column gives additionally the remnant disk mass around the primary star. In the case of Krumholz et al. (2009) and the $480 \mathrm{M}_{\odot}$ case of our own study the $M_{*}$ column gives additionally the remnant disk plus envelope mass. In the case of Yorke \& Sonnhalter (2002) only the simulations with frequency dependent radiation transport are considered. 


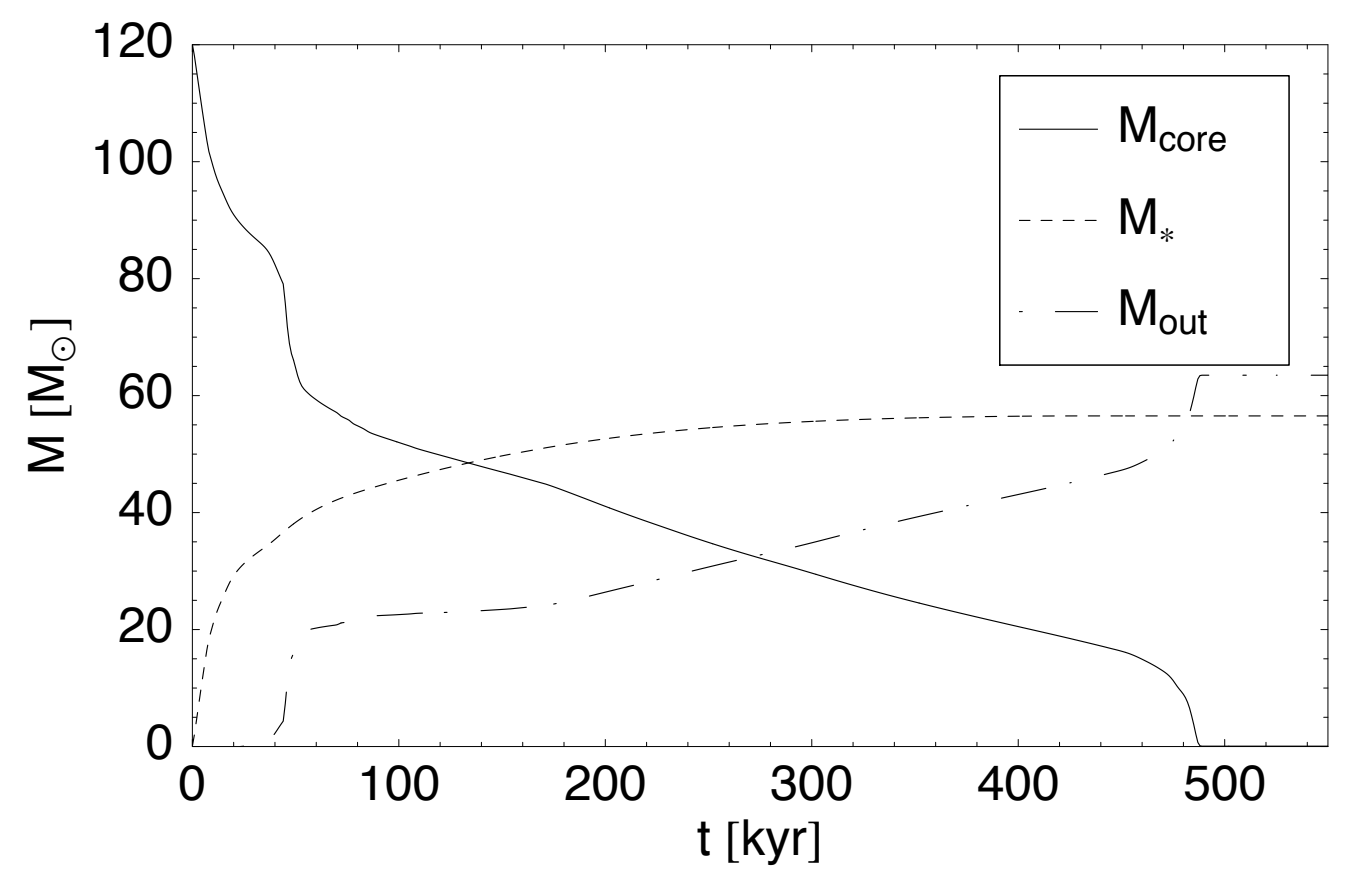

Fig. 20.- : Evolution of mass flows over the inner and outer computational boundary. Solid line: Mass inside the computational domain.

Dashed line: Mass of the forming massive star in the center.

Dot-dashed line: Mass loss due to the radiation pressure in the radially outward direction. 
animation is available in the online-materials.

\subsection{Limitations of our approach}

A minor flaw of our studies of the radiation hydrodynamics around the dust sublimation front is the usage of a simple constant gas opacity of $\kappa_{\text {gas }}=0.01 \mathrm{~cm}^{2} \mathrm{~g}^{-1}$ for the completely evaporated regions around the forming star. In other words, the neighborhood of the star remains optically thin for the stellar irradiation up to the dust sublimation front. From our results of the parameter scan of the inner sink cell radius, we conclude that the prior absorption of the stellar irradiation in dust-free, but potentially optically thick regions, would even enhance the crucial anisotropy of the radiation field detected in our simulations. In this sense, the ignorance of the detailed optical properties of the gas phase does not imply any loss of generality.

The usage of a central sink cell of a specific radius implies further assumptions, e.g. we assume that the mass flow into the central sink cell is accreted by the central star. If, e.g. the mass flow is transferred into an outflow or jet in this inner region near the stellar surface, the stellar growth would be decreased and the final masses of the stars given here represent upper mass limits.

Due to the fact that higher accretion rates lead first to a delay of the star's approach to the main sequence and secondly to a higher ratio of accretion luminosity to stellar luminosity (which increases the importance of the correct knowledge of the stellar radius), the details of the treatment of stellar evolution become important especially in the case of the highest mass cores studied. An even more realistic approach than the usage of tabulated tracks could be achieved by including a stellar evolution code such as Hosokawa \& Omukai (2009) to calculate the ongoing stellar physics in the sink cell consistently in time.

To mimic the effect of angular momentum transport by evolving instabilities in the accretion disk, we made use of the $\alpha$-viscosity model by Shakura \& Sunyaev (1973). Nevertheless, all our code development (radiation transport and self-gravity) and model setup is already in three-dimensional formulation. Hence we started to expand our simulations into full 3D. First, this will allow to compute the angular momentum transport (by gravitational torques) consistently with the formation

and evolution of the accretion disk. Secondly, although we will not study the fragmentation of the outer core regions, the stability or potential fragmentation of the forming massive circumstellar disk can be addressed.

\section{Summary}

We performed high-resolution radiation hydrodynamics simulations of monolithic pre-stellar core collapses including frequency dependent radiative feedback. A broad parameter space of various numerical configurations and initial conditions was scanned. The dust sublimation front in 


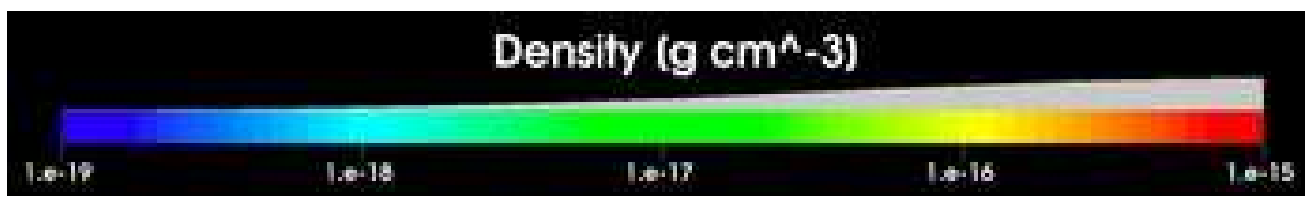

(a) Color scale of the gas density, plotted in logarithmic scale from $10^{-19}$ up to $10^{-15} \mathrm{~g} \mathrm{~cm}^{-3}$.

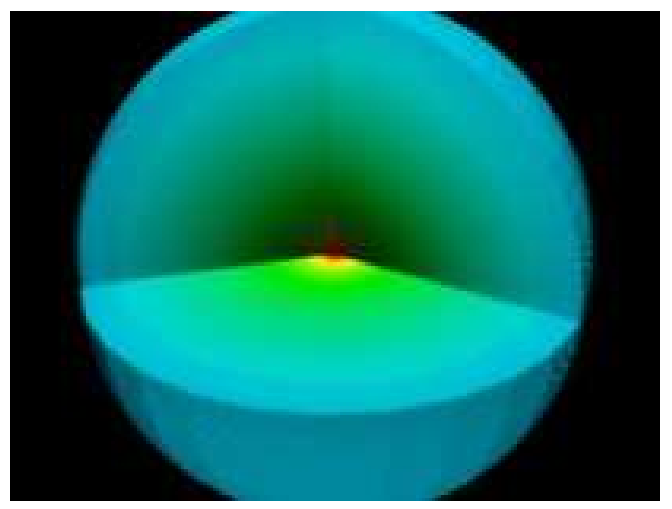

(b) 0 kyr

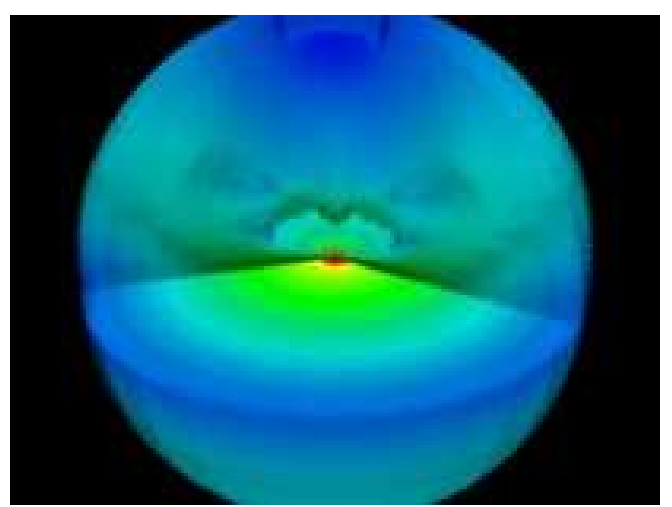

(d) $40 \mathrm{kyr}$

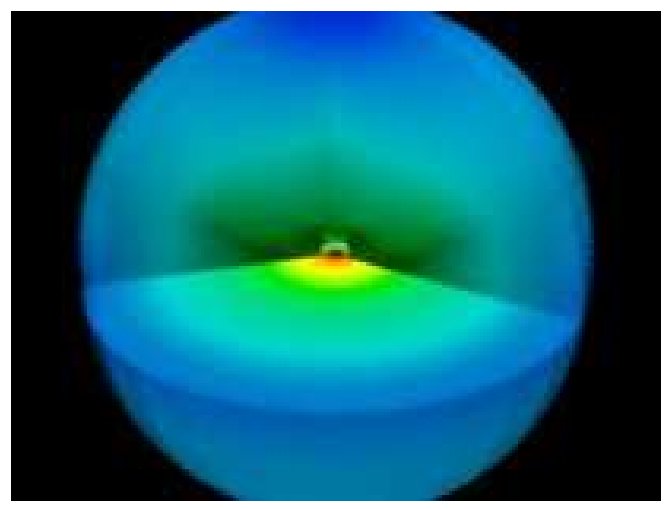

(c) $30 \mathrm{kyr}$

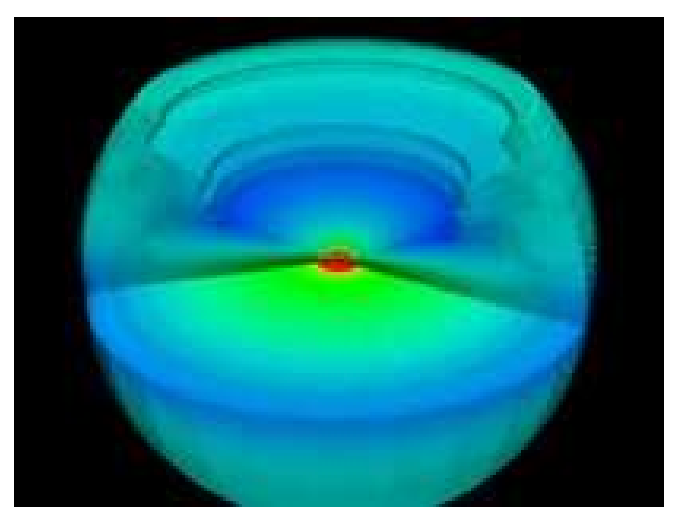

(e) $50 \mathrm{kyr}$

Fig. 21.- : Simulation snapshots from a collapse of a $120 \mathrm{M}_{\odot}$ pre-stellar core. All images show the same scale of the whole pre-stellar core with an initial diameter of $0.2 \mathrm{pc}$. 


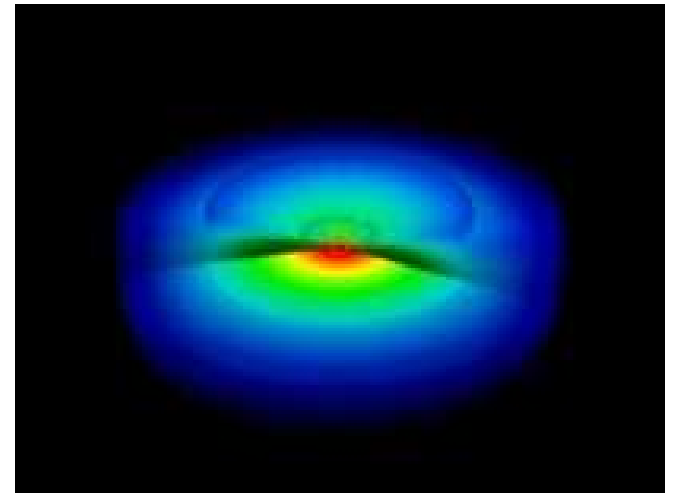

(f) $200 \mathrm{kyr}$

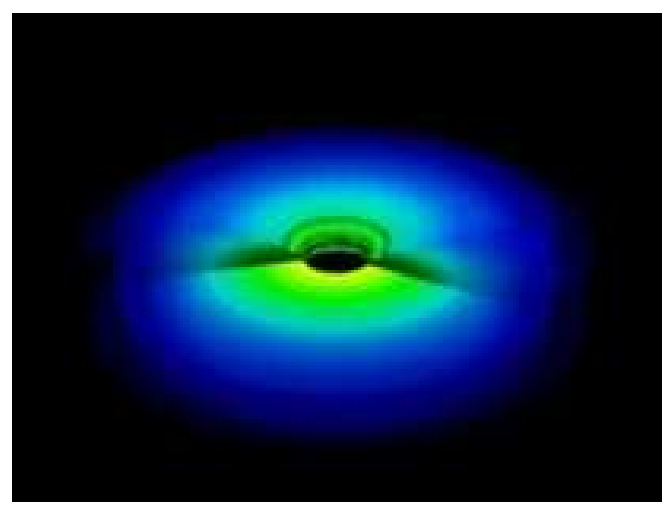

(h) $450 \mathrm{kyr}$

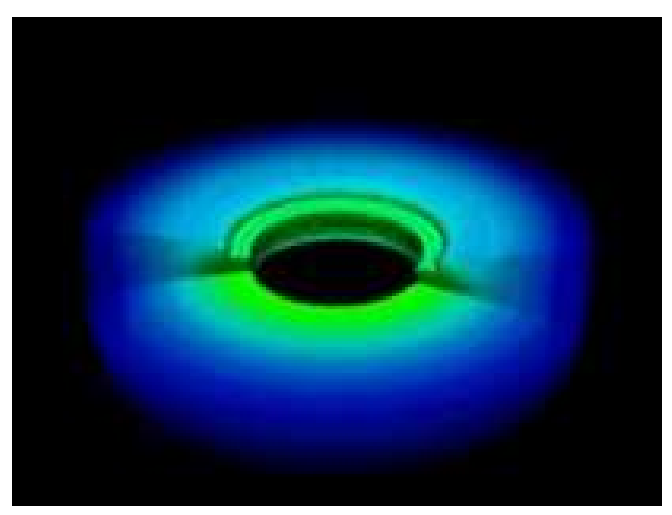

(j) $470 \mathrm{kyr}$

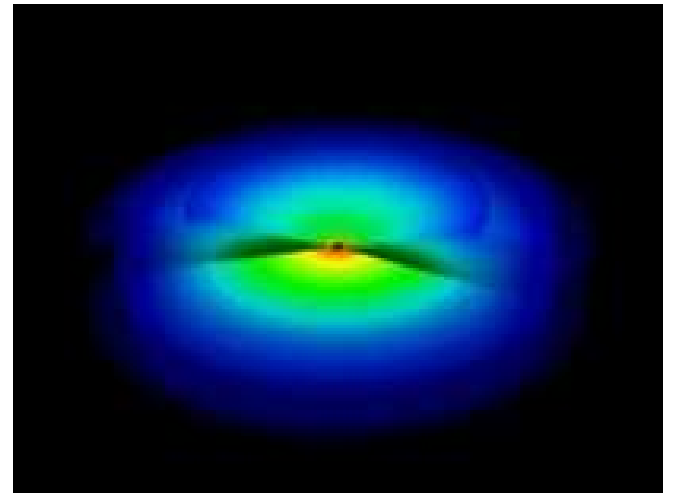

(g) $400 \mathrm{kyr}$

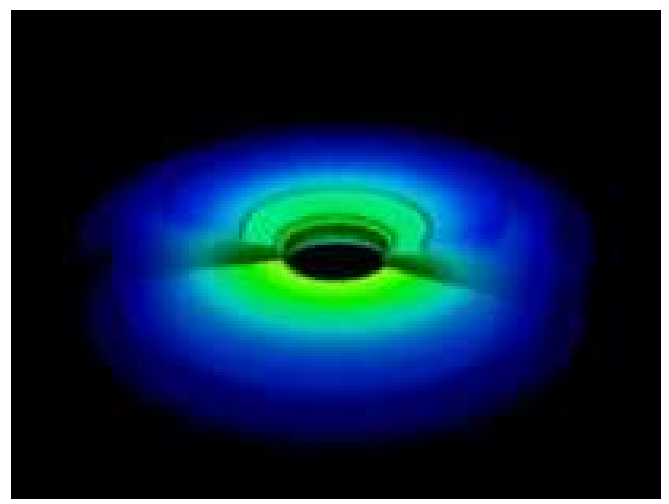

(i) $460 \mathrm{kyr}$

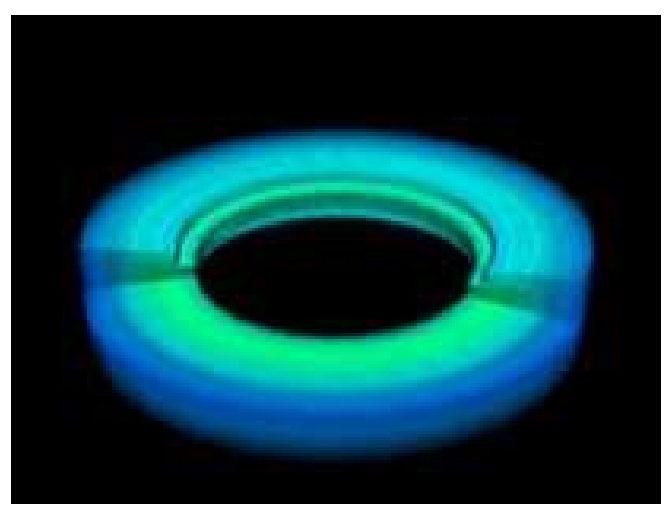

(k) $480 \mathrm{kyr}$

Fig. 21.-: Continuation of Fig. 21 
the vicinity of the forming star could be resolved down to $1.27 \mathrm{AU}$. The evolution of the system was computed over its whole accretion phase of several $10^{5} \mathrm{yr}$. The usage of frequency dependent ray-tracing in our newly developed radiation module denotes the most realistic radiation transport method used in multi-dimensional hydrodynamic simulations of massive star formation by now. The broad parameter studies, especially regarding the size of the sink cell and the initial core mass, reveal new insights of the radiative feedback onto the accretion flow during the formation of a massive star:

In the case of spherically symmetric accretion flows, we confirm the results of previous research studies (Larson \& Starrfield 1971; Kahn 1974; Yorke \& Krügel 1977; Wolfire \& Cassinelli 1987) that the thermal radiation pressure by re-emitted photons behind the dust sublimation front overcomes gravity, stops the accretion flow, and finally reverts the in-falling envelope. The upper mass limit of spherically symmetric accretion for our specific dust (Laor \& Draine 1993) and stellar evolution model (Hosokawa \& Omukai 2009) constrains the final stellar mass to be less than $40 \mathrm{M}_{\odot}$.

In the case of disk accretion, the thermal radiation field generates a strong anisotropic feature, similar to the flashlight effect, which focus lies on the escape of radiation through optically thin cavities. We found that it is strict necessary to include the dust sublimation front in the computational domain, to reveal the persistent anisotropy during the long-term evolution of the accretion disk. This requirement as well as a steady feeding of the accretion disk from the outer core regions maintain the anisotropic structure of the thermal radiation field. The short accretion phases of the disks in the simulations by Yorke \& Sonnhalter (2002) are a result of the fact that they did not include the dust sublimation front in their simulations, as clearly shown in our result of the parameter scan of the size of the central sink cell (see Sect. 5.1). Additional feeding of the disk by unstable outflow regions, as stated in Krumholz et al. (2009), would enhance this anisotropy but is not necessary. As a consequence, we conclude that the radiation pressure problem in the formation of massive stars can be reduced to the question if the non-spherically symmetric stellar environment is dense or opaque enough to generate a strong anisotropy of the thermal radiation field.

These mechanisms allow the central star to increase its mass far beyond the upper mass limit found in the case of spherical accretion flows. For an initial mass of the pre-stellar host core of $60,120,240$, and $480 \mathrm{M}_{\odot}$ the masses of the final stars formed in our simulations of the disk accretion scenario add up to $28.2,56.5,92.6$, and at least $137.2 \mathrm{M}_{\odot}$ respectively. Indeed, the final massive stars are the most massive stars ever formed in a multi-dimensional radiation hydrodynamics simulation so far.

This research has been supported by the International Max-Planck Research School for Astronomy and Cosmic Physics at the University of Heidelberg (IMPRS-HD). Author H. Klahr has been supported in part by the Deutsche Forschungsgemeinschaft (DFG) through grant DFG Forschergruppe 759 "The Formation of Planets: The Critical First Growth Phase". Author H. Beuther acknowledges financial support by the Emmy-Noether-Programm of the DFG through grant BE2578. 
Many of our simulations scanning the broad parameter space have been performed on the rio as well as on the pia cluster of the Max Planck computing center in Garching. We acknowledge Andrea Mignone, the main developer of the open source magneto-hydrodynamics code Pluto, as well as Petros Tzeferacos, who implemented the viscosity tensor into Pluto. Author R. Kuiper thanks especially Cornelis P. Dullemond, Mario Flock, Johannes Schönke, and Takashi Hosokawa for their contributions. Furthermore, R. Kuiper thank Harold Yorke, Neal Turner, Jürgen Steinacker, Benoit Commercon, Bhargav Vaidya, and Cassandra Fallscheer for fruitful discussions.

\section{A. Parameter scans of the resolution}

Numerical hydrodynamics simulations involve a discretization of the underlying equations of hydrodynamics given in continuous space, cp. Eqs. (2)-(4). This causes a discretization error, which in general vanishes for infinitely high resolution of the numerical solver method. To compute a specific quantity, such as the accretion history, with a specific accuracy therefore needs a specific resolution, which is necessary to damp the discretization errors down to the requested accuracy. In this way it is possible to guarantee the achievement of a converged result. Although this procedure is a must in numerical research to achieve reliable results, the overhead of cpu time needed for convergence runs inhibits their realization in most present-day astronomical simulations, especially in multi-dimensional radiation hydrodynamics, which are performed almost at the upper limit of the computational power of the available clusters. To fix the number of grid cells, which are necessary for a correct representation of the radiation fluid interactions, we perform several simulations with

varying resolution. Focusing on the inner regions of the pre-stellar core, the radial cell sizes of the grid thereby grow logarithmically from inside out as described in Sect. 2.1.

\section{A.1. 1D convergence runs}

\section{A.1.1. Simulations}

The initial conditions and numerical parameters of these convergence runs are described in Sect. 3. The simulations are performed for an initial core mass of $M_{\text {core }}=60 \mathrm{M}_{\odot}$ and with an inner sink cell radius of $r_{\min }=1 \mathrm{AU}$. We follow the long-term evolution of the system for at least 163 kyr, representing 2.4 free fall times of the pre-stellar core. The resulting mass growth $M_{*}(t)$ of the centrally forming star is displayed in Fig. 22.

\section{A.1.2. Conclusions}

The lowest resolution run $\left(N_{r}=32\right)$ fails to compute the correct amount of accretion already during the mostly isothermal initial free fall phase (up to $25 \mathrm{kyr}$ ). For higher resolution runs 


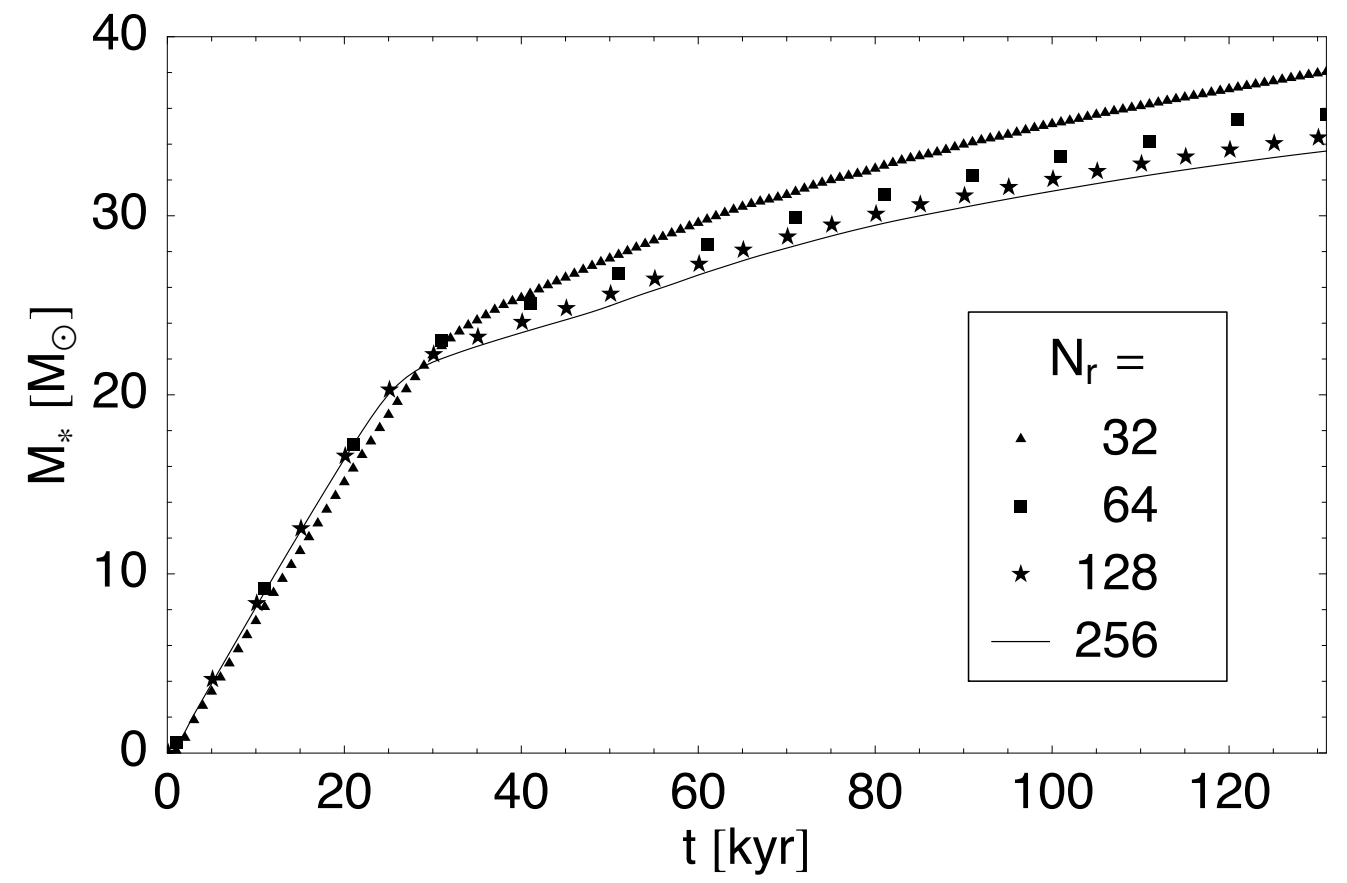

Fig. 22.- : Stellar mass $M_{*}$ as a function of time $t$ for four different resolutions of the spherically symmetric pre-stellar core collapse simulations. The number of grid cells $N_{r}$ varies from 32 to 256 , corresponding to a size of the smallest grid cell of $(\Delta r)_{\min }=0.36$ AU to 0.04 AU respectively. 
with 64 grid cells or more, the mass growth of the forming star is identical during this phase. At a later evolutionary epoch, when radiative feedback becomes important, simulations with higher resolution lead generally to a slower mass growth. The deviation of a specific run to the next one with double resolution shrinks towards higher resolution, so the simulations fulfill the requirement of a monotonic convergence. Our one-dimensional simulations with varying initial core masses (Sect. 4.2) use 128 grid cells in the radial direction and an inner radial boundary at $r_{\min }=1 \mathrm{AU}$ corresponding to a grid size of $(\Delta r)_{\min }=0.08 \mathrm{AU}$ for the innermost grid cell.

\section{A.2. 2D convergence runs}

\section{A.2.1. Simulations}

To fix the number of grid cells necessary for computing the correct physics of the radiation fluid interaction, we performed several simulations with varying resolution in the two-dimensional setup, too. The basic initial conditions and numerical parameter used for these convergence runs are described in Sect. 3. The simulations are performed for a core mass of $M_{\text {core }}=60 \mathrm{M}_{\odot}$ and the inner boundary of the computational domain is located at $r_{\min }=10 \mathrm{AU}$. We followed the evolution of the collapsing core up to $33 \mathrm{kyr}$ ( 0.5 free fall times) for the highest resolution case yet and up to several hundred kyr (about 10 free fall times) for a long-term convergence run. The accretion history and the corresponding mass growth of the centrally forming star are displayed in Figs. 23 and 24 .

\section{A.2.2. Conclusions}

In contrast to the purely one-dimensional in-fall (Sect. A.1), the centrifugal forces slow down the radially proceeding dynamics. So the usage of 64 grid cells in the radial direction, corresponding to a radial grid size of the innermost cells of $(\Delta r)_{\min }=1.27 \mathrm{AU}$, yields a converged result for the accretion rate onto the forming high-mass star. The low-resolution run with only 32 grid cells in the radial direction clearly fails to compute the correct onset of disk formation after 8 kyr. Due to the clear dominance of the motion of gas in the radial direction during the initial 'free fall' phase up to roughly 8 kyr the accretion rates of this epoch are independent of the resolution used in the polar direction. The required resolution in the polar direction to compute a converged result also during later epochs remains notably poor, reflecting the fact that the complex radiation hydrodynamics interactions act mostly in the radial direction. This result confirms the expedient choice of spherical coordinates in monolithic core collapse simulations. Higher resolution of the polar stratification of the forming circumstellar disk mostly influences the cooling of the irradiated and viscously heated midplane layer. The usage of only 4 or 8 grid cells in the polar direction therefore results in stronger fluctuations of the accretion flow, which vanish in the higher resolution runs (clearly visible in the lower panel of Fig. 23). On the other hand the runs with low resolution in the polar direction 

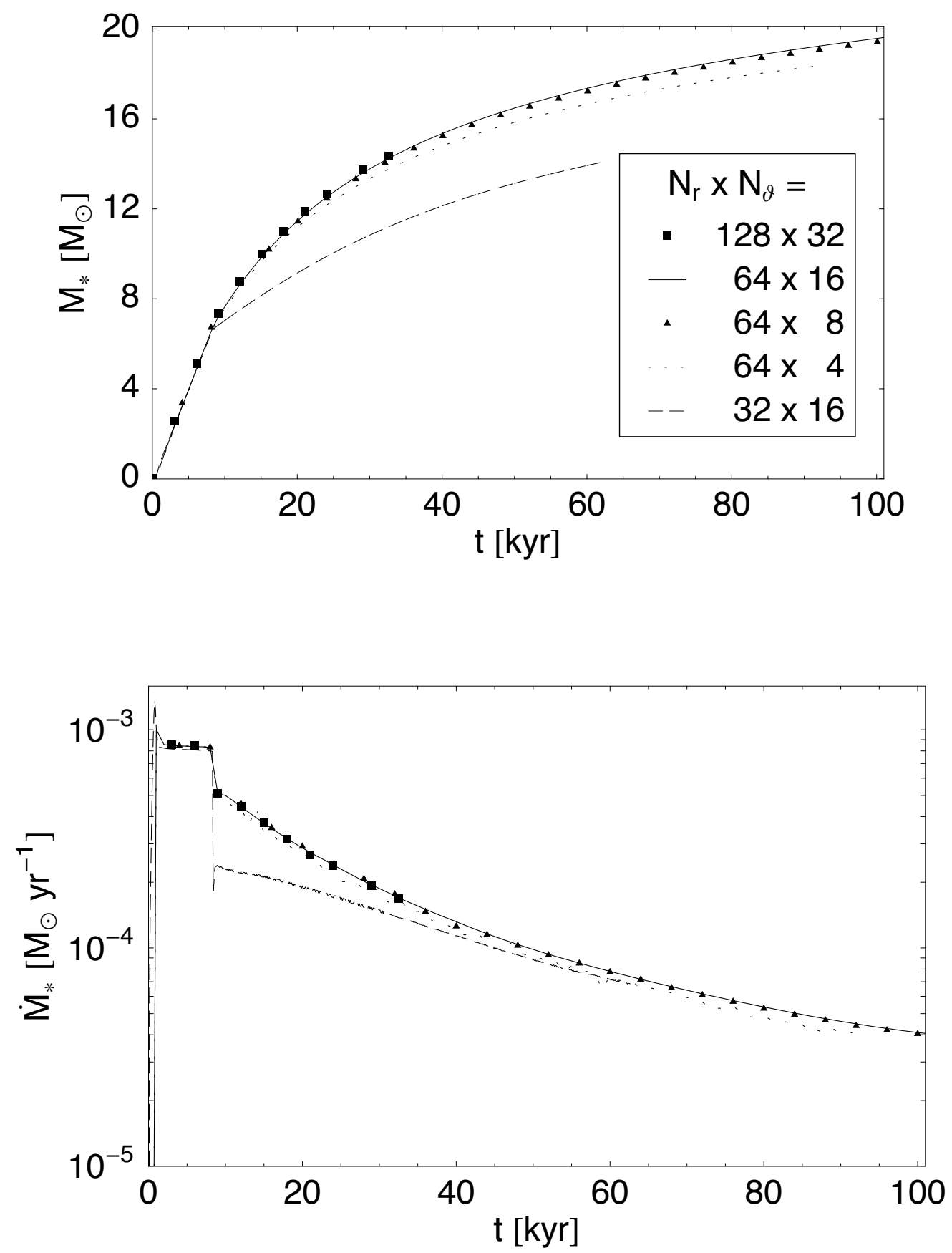

Fig. 23.- : Stellar mass $M_{*}$ (upper panel) and accretion rate $\dot{M}_{*}$ (lower panel) as a function of time $t$ for five different resolution to determine the adequate number of grid cells necessary for the collapse simulations of the rotating pre-stellar cores. 

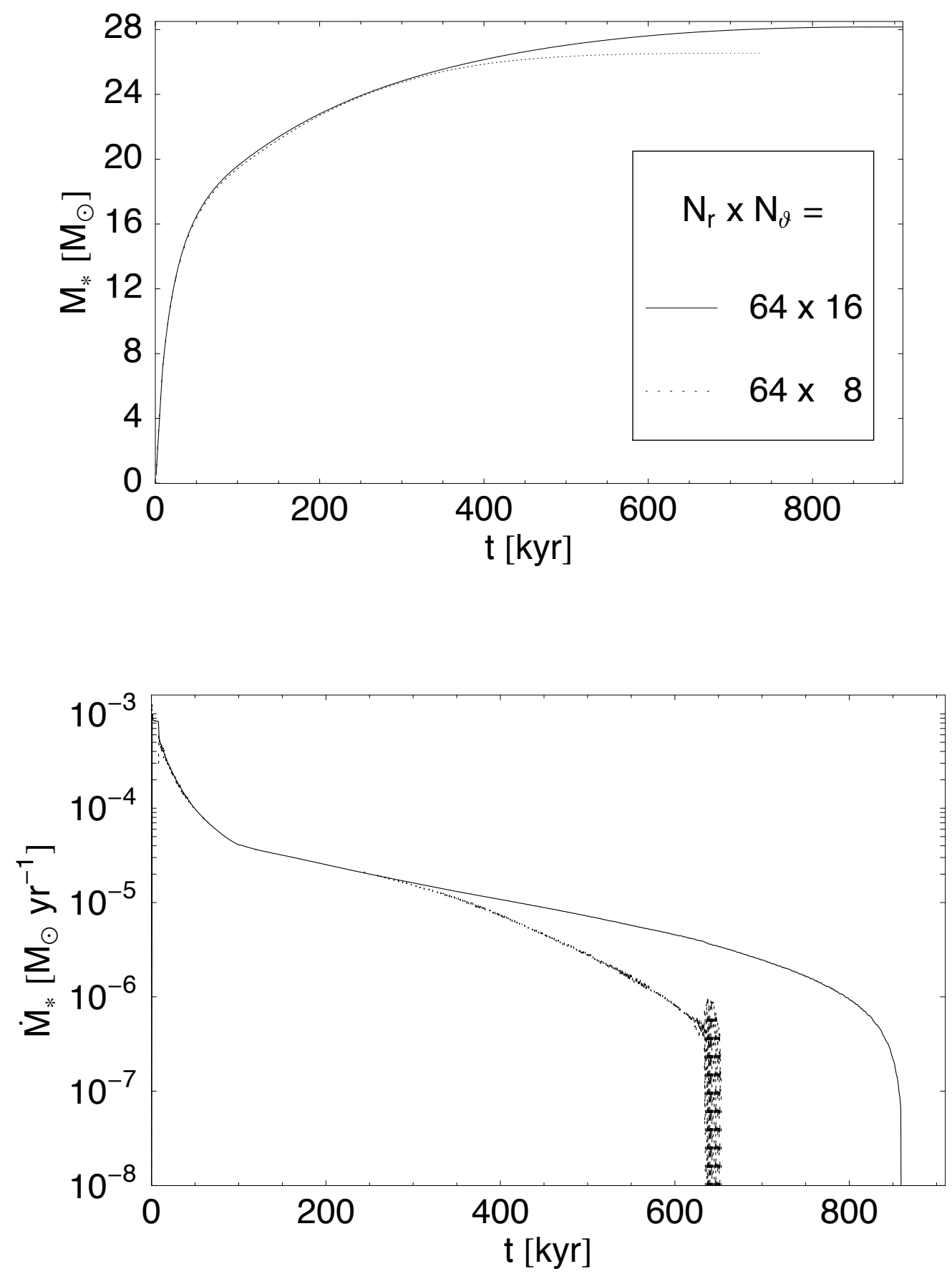

Fig. 24.-: Stellar mass $M_{*}$ (upper panel) and accretion rate $\dot{M}_{*}$ (lower panel) as a function of time $t$ for two different resolution in a long-term convergence study up to the end of the disk accretion phase. 
underestimate the mass growth of the forming star only slightly (upper panel in Fig. 23). The deviations of each run to the next run in higher resolution shrink towards higher resolution, that means the simulation series yields a monotonous convergence. The long-term convergence study (Fig. 24) clearly shows that the point in time when the disk looses its shadowing property depends on the polar resolution of the circumstellar disk. Higher resolution of the disk's stratification results in a stronger anisotropy of the thermal radiation field and therefore minimizes the radiation pressure on the accretion flow.

The runs with $64 \times 16$ and $128 \times 32$ grid cells show fully converged results even during the epoch of the most rapid changes at the onset of disk formation at $8 \mathrm{kyr}$. The spike in the accretion rate downwards during this onset represents the short period in time, in which for the first time a fluid package from the outer core region arrived at the innermost radius $r_{\min }$ with high enough angular momentum to compensate the stellar gravity. Quickly hereafter the following mass builds up a circumstellar disk, in which the shear viscosity yields an angular momentum transfer outwards resulting in a steady accretion rate anew. At later evolutionary phases the amplitude of the accretion rate is mostly a result of a quasi-stationary accretion flow inwards and an interactive radiative flux in the outward direction, which smoothly grows proportional to the luminosity of the forming massive star. The deviations of the individual runs during this more evolved and 'less violent' phase shrink again for all resolutions studied. Our two-dimensional simulations presented use $64 \times 16$ grid cells as the default setup corresponding to a grid size of $(\Delta r \times r \Delta \theta)_{\min }=1.27 \mathrm{AU}$ x 1.04 AU for the innermost grid cells.

\section{REFERENCES}

Balbus, S. A. 2003, ARA\&A, 41, 555

Balbus, S. A. \& Hawley, J. F. 1991, ApJ, 376, 214

Bate, M. R. 2009a, MNRAS, 392, 1363

-. 2009b, MNRAS, 392, 590

Beltrán, M. T., Cesaroni, R., Neri, R., Codella, C., Furuya, R. S., Testi, L., \& Olmi, L. 2004, ApJ, 601, L187

-. 2005, A\&A, 435, 901

Beuther, H., Churchwell, E. B., McKee, C. F., \& Tan, J. C. 2007, in: Protostars and Planets V, ed. B. Reipurth, D. Jewitt, \& K. Keil, 165

Beuther, H. \& Shepherd, D. 2005, in: Cores to Clusters: Star Formation with Next Generation Telescopes, ed. M. S. Nanda Kumar, 105

Beuther, H., Sridharan, T. K., \& Saito, M. 2005a, ApJ, 634, L185 
Beuther, H. \& Walsh, A. J. 2008, ApJ, 673, L55

Beuther, H., Walsh, A. J., \& Longmore, S. N. 2009, The Astrophysical Journal Supplement, 184, 366

Beuther, H., Zhang, Q., Sridharan, T. K., \& Chen, Y. 2005b, ApJ, 628, 800

Birkmann, S. M., Krause, O., Hennemann, M., Henning, T., Steinacker, J., \& Lemke, D. 2007, A\&A, 474, 883

Black, D. C. \& Bodenheimer, P. 1975, ApJ, 199, 619

Bodenheimer, P. 1995, ARA\&A, 33, 199

Boley, A. C., Durisen, R. H., Nordlund, Å., \& Lord, J. 2007, ApJ, 665, 1254

Bonnell, I. A. \& Bate, M. R. 2002, MNRAS, 336, 659

Bonnell, I. A., Bate, M. R., \& Zinnecker, H. 1998, MNRAS, 298, 93

Bonnell, I. A., Vine, S. G., \& Bate, M. R. 2004, MNRAS, 349, 735

Cesaroni, R., Felli, M., Testi, L., Walmsley, C. M., \& Olmi, L. 1997, A\&A, 325, 725

Cesaroni, R., Neri, R., Olmi, L., Testi, L., Walmsley, C. M., \& Hofner, P. 2005, A\&A, 434, 1039

Chini, R., Henning, T., \& Pfau, W. 1991, A\&A, 247, 157

Davis, C. J., Moriarty-Schieven, G., Eislöffel, J., Hoare, M. G., \& Ray, T. P. 1998, AJ, 115, 1118

Duschl, W. J., Strittmatter, P. A., \& Biermann, P. L. 2000, A\&A, 357, 1123

Edgar, R. \& Clarke, C. 2003, MNRAS, 338, 962

—. 2004, MNRAS, 349, 678

Fallscheer, C., Beuther, H., Zhang, Q., Keto, E., \& Sridharan, T. K. 2009, A\&A, 504, 127

Gammie, C. F. 2001, ApJ, 553, 174

Harvey, P. M., Campbell, M. F., Hoffmann, W. F., Thronson, H. A., \& Gatley, I. 1979, ApJ, 229, 990

Hawley, J. F. \& Balbus, S. A. 1991, ApJ, 376, 223

Henning, T., Lapinov, A., Schreyer, K., Stecklum, B., \& Zinchenko, I. 2000, A\&A, 364, 613

Ho, P. T. P. \& Haschick, A. D. 1986, ApJ, 304, 501

Hosokawa, T. \& Omukai, K. 2009, ApJ, 691, 823 
Isella, A. \& Natta, A. 2005, A\&A, 438, 899

Jijina, J. \& Adams, F. C. 1996, ApJ, 462, 874

Kahn, F. D. 1974, A\&A, 37, 149

Keto, E. R. 2003, ApJ, 599, 1196

Keto, E. R., Ho, P. T. P., \& Reid, M. J. 1987, ApJ, 323, L117

Klahr, H. H. \& Bodenheimer, P. 2003, ApJ, 582, 869

Krumholz, M. R. 2005, PhD Thesis, 15

Krumholz, M. R., Klein, R. I., \& McKee, C. F. 2007, ApJ, 656, 959

Krumholz, M. R., Klein, R. I., McKee, C. F., Offner, S. S. R., \& Cunningham, A. J. 2009, Science, 323,754

Krumholz, M. R., McKee, C. F., \& Klein, R. I. 2005, ApJ, 618, L33

Kuiper, R., Klahr, H., Dullemond, C., Kley, W., \& Henning, T. 2010, A\&A, 511, 81

Landau, L. D. \& Lifshitz, E. M. 1987, Pergamon Press, Oxford, 552

Laor, A. \& Draine, B. T. 1993, ApJ, 402, 441

Larson, R. B. \& Starrfield, S. 1971, A\&A, 13, 190

Laughlin, G. P. \& Bodenheimer, P. 1994, ApJ, 436, 335

Lyder, D. A., Belton, D. S., \& Gower, A. C. 1998, AJ, 116, 840

McKee, C. F. \& Ostriker, E. C. 2007, ARA\&A, 45, 565

McKee, C. F. \& Tan, J. C. 2003, ApJ, 585, 850

Mignone, A., Bodo, G., Massaglia, S., Matsakos, T., Tesileanu, O., Zanni, C., \& Ferrari, A. 2007, ApJS, 170, 228

Nakano, T. 1989, ApJ, 345, 464

Ossenkopf, V. \& Henning, T. 1994, A\&A, 291, 943

Pascucci, I., Wolf, S., Steinacker, J., Dullemond, C. P., Henning, T., Niccolini, G., Woitke, P., \& Lopez, B. 2004, A\&A, 417, 793

Pollack, J. B., Hollenbach, D., Beckwith, S., Simonelli, D. P., Roush, T., \& Fong, W. 1994, ApJ, 421,615 
Schreyer, K., Henning, T., van der Tak, F. F. S., Boonman, A. M. S., \& van Dishoeck, E. F. 2002, A\&A, 394, 561

Schreyer, K., Semenov, D., Henning, T., \& Forbrich, J. 2006, ApJ, 637, L129

Shakura, N. I. \& Sunyaev, R. A. 1973, A\&A, 24, 337

Shu, F. H., Lizano, S., \& Adams, F. C. 1987, in: Star forming regions, ed. M. Peimbert, J. Jugaku, 115,417

Strang, G. 1968, SIAM Journal on Numerical Analysis, 5, 506

Torrelles, J. M., Ho, P. T. P., Moran, J. M., Rodriguez, L. F., \& Canto, J. 1986, ApJ, 307, 787

Truelove, J. K., Klein, R. I., McKee, C. F., Holliman, J. H., Howell, L. H., \& Greenough, J. A. 1997, ApJL, 489, L179

Vaidya, B., Fendt, C., \& Beuther, H. 2009, ApJ, 702, 567

van Leer, B. 1979, JCP, 32, 101

Whitney, B. A. 2005, Nat, 437, 37

Wolfire, M. G. \& Cassinelli, J. P. 1987, ApJ, 319, 850

Wu, Y., Zhang, Q., Chen, H.-R., Yang, C., Wei, Y., \& Ho, P. T. P. 2005, AJ, 129, 330

Yang, S., Durisen, R. H., Cohl, H. S., Imamura, J. N., \& Toman, J. 1991, Icarus, 91, 14

Yorke, H. W., Bodenheimer, P., \& Laughlin, G. P. 1995, ApJ, 443, 199

Yorke, H. W. \& Krügel, E. 1977, A\&A, 54, 183

Yorke, H. W. \& Sonnhalter, C. 2002, ApJ, 569, 846

Zhang, Q. \& Ho, P. T. P. 1997, ApJ, 488, 241

Zhang, Q., Hunter, T. R., Beuther, H., Sridharan, T. K., Liu, S.-Y., Su, Y.-N., Chen, H.-R., \& Chen, Y. 2007, ApJ, 658, 1152

Zhang, Q., Hunter, T. R., \& Sridharan, T. K. 1998, ApJ, 505, L151

Zhang, Q., Hunter, T. R., Sridharan, T. K., \& Ho, P. T. P. 2002, ApJ, 566, 982

Zinnecker, H. \& Yorke, H. W. 2007, ARA\&A, 45, 481 
